CTP-TAMU-47/95

hep-th/9512012

\title{
p-brane Solitons in Maximal Supergravities
}

\author{
H. Lü and C.N. Pope \\ Center for Theoretical Physics, Texas A\&M University, College Station, Texas 77843
}

\begin{abstract}
$\underline{\text { ABSTRACT }}$
In this paper, we give a construction of $p$-brane solitons in all maximal supergravity theories in $4 \leq D \leq 11$ dimensions that are obtainable from $D=11$ supergravity by dimensional reduction. We first obtain the full bosonic Lagrangians for all these theories in a formalism adapted to the $p$-brane soliton construction. The solutions that we consider involve one dilaton field and one antisymmetric tensor field strength, which are in general linear combinations of the basic fields of the supergravity theories. We also study the supersymmetry properties of the solutions by calculating the eigenvalues of the Bogomol'nyi matrices, which are derived from the commutators of the supercharges. We give an exhaustive list of the supersymmetric $p$-brane solutions using field strengths of all degrees $n=4,3,2,1$, and the non-supersymmetric solutions for $n=4,3,2$. As well as studying elementary and solitonic solutions, we also discuss dyonic solutions in $D=6$ and $D=4$. In particular, we find that the Bogomol'nyi matrices for the supersymmetric massless dyonic solutions have indefinite signature.
\end{abstract}

Research supported in part by DOE Grant DE-FG05-91-ER40633 


\section{Introduction}

Recent progress in the understanding of duality and the non-perturbative structure of string theories has emphasised the importance of solitonic string and $p$-brane solutions in the low-energy effective actions. It is therefore of interest to attempt to classify all such solutions. Our goal in this paper is to study $p$-brane solitons in all the maximal supergravities related to 11-dimensional supergravity or, equivalently, type IIA supergravity in 10 dimensions, by dimensional reduction. Starting from the 11-dimensional theory, whose bosonic sector comprises the vielbein and a 4-index antisymmetric tensor field strength, one obtains theories by dimensional reduction containing a vielbein, dilatonic scalar fields, and various $n$-index antisymmetric tensor field strengths $F_{n}$ with $n=1,2,3,4$. The $p$ brane solutions we shall be considering are of the kind discussed in [1-9], and involve non-vanishing background values for the vielbein, a dilatonic scalar, and an $n$-index field strength. The dilatonic scalar and the field strength, which we shall refer to as the canonical dilaton and field strength, may be linear combinations of the original fields in the supergravity theory. The relevant part of the $D$-dimensional Lagrangian is given by

$$
\mathcal{L}=e R-\frac{1}{2} e(\partial \phi)^{2}-\frac{1}{2 n !} e e^{a \phi} F_{n}^{2} .
$$

We shall be focusing on isotropic $p$-brane solutions, for which the metric ansatz is given by

$$
d s^{2}=e^{2 A} d x^{\mu} d x^{\nu} \eta_{\mu \nu}+e^{2 B} d y^{m} d y^{m},
$$

where $x^{\mu}(\mu=0, \ldots, d-1)$ are the coordinates of the $(d-1)$-brane world volume, and $y^{m}$ are the coordinates of the $(D-d)$-dimensional tranverse space. The functions $A$ and $B$, as well as the dilaton $\phi$, depend only on $r=\sqrt{y^{m} y^{m}}$. Thus the ansatz preserves an $S O(1, d-1) \times S O(D-d)$ subgroup of the original $S O(1, D-1)$ Lorentz group. The constant $a$ in the dilaton prefactor can be parametrised as [9]

$$
a^{2}=\Delta-\frac{2 d \tilde{d}}{D-2}
$$

where $\tilde{d} \equiv D-d-2$ and $d \tilde{d}=(n-1)(D-n-1)$. In $D=11$, the absence of a dilaton implies that $\Delta=4$. In fact this value occurs in the dilaton prefactors for all field strengths obtained simply by Kaluza-Klein dimensional reduction, since the reduction procedure always preserves the value of $\Delta[9]$. However, if the field strength $F_{n}$ used in a particular $p$-brane solution is formed from a linear combination of these original field strengths, then it will have a dilaton prefactor which, after setting the non-participating fields to zero, has $\Delta<4$.

For each $F_{n}$ with $n \geq 2$, there are two different ansätze that also preserve the same subgroup, namely [1, 4]

$$
F_{r \mu_{1} \ldots \mu_{n-1}}=\epsilon_{\mu_{1} \ldots \mu_{n-1}}\left(e^{C}\right)^{\prime} \quad \text { or } \quad F_{m_{1} \ldots m_{n}}=\lambda \epsilon_{m_{1} \ldots m_{n} p} \frac{y^{p}}{r^{n+1}}
$$


where a prime denotes a derivative with respect to $r$. The first case gives rise to an elementary $(d-1)$-brane with $d=n-1$, and the second gives rise to a solitonic $(d-1)$-brane with $d=D-n-1$. The solutions are given by [9]

$$
\begin{aligned}
d s^{2} & =\left(1+\frac{k}{r \tilde{d}}\right)^{-\frac{4 \tilde{d}}{\Delta(D-2)}} d x^{\mu} d x^{\nu} \eta_{\mu \nu}+\left(1+\frac{k}{r \tilde{d}}\right)^{\frac{4 d}{\Delta(D-2)}} d y^{m} d y^{m} \\
e^{\phi} & =\left(1+\frac{k}{r \tilde{d}}\right)^{\frac{2 a}{\epsilon \Delta}}
\end{aligned}
$$

where $\epsilon=1$ and -1 for the elementary and the solitonic solutions respectively, and $k=-\sqrt{\Delta} \lambda /(2 \tilde{d})$. In the elementary case, the function $C$ satisfies the equation

$$
e^{C}=\frac{2}{\sqrt{\Delta}}\left(1+\frac{k}{r \tilde{d}}\right)^{-1} .
$$

Note that the dual of the solution for the field strength in the elementary case is identical to the field strength of the solitonic case, and vice versa. For this reason, we shall only consider solutions for field strengths with $n \leq D / 2$.

So far we have discussed the solutions (1.5) for an $n$-form field strength with $n>1$. When $n=1$, and hence $\tilde{d}=0$, one can only construct a solitonic solution, given by eqn. (1.5) with $k r^{-\tilde{d}} \longrightarrow k \log r$ and $\tilde{d} \longrightarrow 0$. The solution (1.5) is also valid when $n=0$, giving rise to a purely dilatonic $(D-2)$-brane in $D$ dimensions 10, 11, which we shall not consider in this paper.

For a given value of $n$, the metrics of the elementary and solitonic $p$-brane solutions (1.5) are characterised completely by the value of $\Delta$. However, as we shall see later, inequivalent fieldstrength configurations can sometimes give rise to the same value of $\Delta$. These solutions, although having the same value of $\Delta$, break different fractions of the $D=11$ supersymmetry. The purpose of this paper is to classify the possible $p$-brane solutions that can arise from a maximal supergravity theory in any dimension $D$ according to their values of $\Delta$ and their field-strength configurations. We shall also examine the supersymmetry of these solutions.

In dimensions $D=2 n$, the field strength $F_{n}$ can in principle use both the elementary and solitonic ansätze (1.4) simultaneously. In this case, the equations of motion reduce to two independent differential equations

$$
\phi^{\prime \prime}-n \frac{\phi^{\prime}}{r}=\frac{1}{2} a\left(S_{1}^{2}-S_{2}^{2}\right), \quad A^{\prime \prime}+n \frac{A^{\prime}}{r}=\frac{1}{4}\left(S_{1}^{2}+S_{2}^{2}\right)
$$

together with the relations $B=-A,\left(e^{C}\right)^{\prime}=\lambda_{1} e^{a \phi+2(n-1) A} r^{-n}$, where $S_{1}$ and $S_{2}$ are given by

$$
S_{1}=\lambda_{1} e^{\frac{1}{2} a \phi+(n-1) A} r^{-n}, \quad S_{2}=\lambda_{2} e^{-\frac{1}{2} a \phi+(n-1) A} r^{-n} .
$$

The equations (1.7) admit a simple solution either when $a^{2}=n-1$, given by

$$
e^{-\frac{1}{2} a \phi-(n-1) A}=1+\frac{\lambda_{1}}{a \sqrt{2}} r^{-n+1}, \quad e^{+\frac{1}{2} a \phi-(n-1) A}=1+\frac{\lambda_{2}}{a \sqrt{2}} r^{-n+1},
$$


or when $a=0$, given by

$$
\phi=0, \quad e^{-(n-1) A}=1+\frac{1}{2} \sqrt{\frac{\lambda_{1}^{2}+\lambda_{2}^{2}}{(n-1)}} r^{-n+1} .
$$

The former solution includes the dyonic strings in $D=6$ that were found in ref. [12], and the latter solution includes the self-dual string in $D=6$ [6]. The dyonic solutions (1.9) and (1.10) apply only to $a^{2}=n-1$ and $a^{2}=0$, and hence $\Delta=2 n-2$ and $\Delta=n-1$, respectively. In $D=8,6$ and 4 dimensions, the values of $\Delta$ are $\{6,3\},\{4,2\}$ and $\{2,1\}$ respectively. Thus such a dyonic solution does not exist in maximal supergravity in $D=8$ since the 4 -form has $\Delta=4$. We shall see in the next section that such a dyonic solution is also excluded by consideration of the full set of field equations of the $D=8$ supergravity theory. Note that the solution (1.9) becomes the standard elementary or solitonic solution (1.5) when $\lambda_{2}=0$ or $\lambda_{1}=0$ respectively. When $\lambda_{1}=\lambda_{2}$, the solutions (1.9) and (1.10) are equivalent.

The paper is organised as follows. In section 2, we obtain the complete bosonic Lagrangian of the maximal supergravities in lower dimensions via Kaluza-Klein dimensional reduction from $D=11$. We do this in a formalism that distinguishes between the dilatonic scalar fields, which appear in exponential prefactors, and the 0 -form potentials for 1-form field strengths. In section 3 , we first discuss the formalism for constructing $p$-brane solutions in the maximal supergravity theories. The supersymmetry properties of these solutions can be examined by constructing the appropriate Nester forms, which arise as the commutators of conserved supercharges. We obtain the Nester forms in lower dimensions from the Nester form in $D=11$, by using the Kaluza-Klein procedure. We can read off the "Bogomol'nyi matrices" from these Nester forms. Zero eigenvalues of the the Bogomol'nyi matrix correspond to unbroken supersymmetries in the corresponding $p$ brane solutions. In sections 4,5 and 6 , we obtain the explicit $p$-brane solutions corresponding to the 4-form, 3-form, 2-form and 1-form field strengths, and we discuss their supersymmetry properties. We present the conclusions in section 7. Details of the dimensionally-reduced bosonic Lagrangians can be found in an appendix.

\section{Kaluza-Klein dimensional reduction}

A convenient way of constructing the maximal supergravity theories in arbitrary dimensions is by starting from $D=11$ supergravity and performing Kaluza-Klein dimensional reduction. Although one can directly reduce from 11 to $D$ dimensions, there are advantages in carrying out the procedure step by step, descending through the dimensions one at a time. In this approach, it is easier to identify which of the scalar fields are "dilatonic," i.e. scalar fields that appear in the Lagrangian via exponential factors, as opposed to those that have constant shift symmetries, which should be 
viewed as 0 -form potentials for 1 -form field strengths. Thus the dimensional reduction procedure consists of an iterative application of the basic one-step reduction from $(D+1)$ to $D$ dimensions. We denote the coordinates of the $(D+1)$-dimensional spacetime by $x^{\hat{M}}=\left(x^{M}, z\right)$, where $z$ is the coordinate of the extra dimension. The vielbein of the $(D+1)$-dimensional spacetime is then given by $\hat{e}^{A}=e^{\alpha \varphi} e^{A}$ and $\hat{e}^{\underline{z}}=e^{-(D-2) \alpha \varphi}(d z+\mathcal{A})$, where $e^{A}$ is the vielbein in $(D+1)$ dimensions. In terms of components, $\hat{e}_{\hat{M}}^{\hat{A}}$ is given by

$$
\begin{gathered}
\hat{e}^{A}{ }_{M}=e^{\alpha \varphi} e^{A}{ }_{M}, \quad \hat{e}^{\underline{z}}{ }_{M}=e^{-(D-2) \alpha \varphi} \mathcal{A}_{M}, \\
\hat{e}^{A}{ }_{z}=0, \quad \hat{e}^{\underline{z}}{ }_{z}=e^{-(D-2) \alpha \varphi},
\end{gathered}
$$

where $e^{A}{ }_{M}, \varphi$ and $\mathcal{A}=\mathcal{A}_{M} d x^{M}$ are taken to be independent of the extra coordinate $z$, and ${ }_{M}$ and $z$ denote world indices whilst $A$ and $\underline{z}$ denote tangent-space indices. The constant $\alpha$ is given by $\alpha^{2}=\frac{1}{2(D-1)(D-2)}$. An $n$-index field strength $\hat{F}_{n}$ in $(D+1)$ decomposes into two $z$-independent field strengths $F_{n}$ and $F_{n-1}$ in $D$ dimensions:

$$
\hat{F}_{n}=F_{n}+F_{n-1} \wedge(d z+\mathcal{A}) .
$$

Note that the $D$-dimensional field strengths are in general all of the form $F=d A+\cdots$, where the dots indicate extra "Chern-Simons" terms involving wedge products of lower degree forms and potentials. Under certain circumstances, these modifications to the field strengths will lead to constraints on the allowable field configurations for $p$-brane solutions; we shall discuss this in more detail later. Under this reduction procedure, a bosonic Lagrangian in $(D+1)$ dimensions of the form

$$
\mathcal{L}=\hat{e} \hat{R}-\frac{1}{2} \hat{e}(\partial \phi)^{2}-\frac{1}{2 n !} \hat{e} e^{\hat{a} \hat{\phi}} \hat{F}_{n}^{2}
$$

becomes

$$
\begin{aligned}
\mathcal{L}= & e R-\frac{1}{2} e(\partial \phi)^{2}-\frac{1}{2} e(\partial \varphi)^{2}-\frac{1}{4} e e^{-2(D-1) \alpha \phi} \mathcal{F}^{2} \\
& -\frac{1}{2 n !} e e^{-2(n-1) \alpha \varphi+\hat{a} \phi} F_{n}^{2}-\frac{1}{2(n-1) !} e e^{2(D-n) \alpha \varphi+\hat{a} \phi} F_{n-1}^{2},
\end{aligned}
$$

where $\mathcal{F}=d \mathcal{A}$. One can apply this procedure iteratively to reduce the 11-dimensional supergravity to any lower dimension. Note that in each step of dimensional reduction, a new two-form field strength emerges from the metric.

As one descends through the dimensions, the number of field strengths proliferates. The dimensional reduction of the Lagrangian (2.3) to (2.4) described above indicates the pattern of this proliferation. In each step, a new dilatonic scalar appears, and so there will be $(11-D)$ dilatons in $D$ dimensions. Thus in $(D+1)$ dimensions, the dilaton factor of the field strength $F_{n}$ in Lagrangian (2.3) in general takes the form $e^{\vec{a}_{D+1} \cdot \vec{\phi}_{D+1}} \hat{F}_{n}^{2}$, where $\vec{\phi}_{D+1}=\left(\phi_{1}, \phi_{2}, \ldots, \phi_{10-D}\right)$. Since there is no 
dilaton in 11-dimensional supergravity, we see that $\vec{a}_{11}=0$. It follows from eqn. (2.4) that we obtain the lower dimensional vectors $\vec{a}$ by the following algorithm:

$$
\vec{a}_{D}=\left(\vec{a}_{D+1}, x \sqrt{\frac{2}{(D-1)(D-2)}}\right) \quad \text { with } \quad x= \begin{cases}-(n-1), & \text { for } \hat{F}_{n} \rightarrow F_{n}, \\ (D-n), & \text { for } \hat{F}_{n} \rightarrow F_{n-1}, \\ -(D-1), & \text { for } \mathcal{F} .\end{cases}
$$

Here the three cases refer to an $n$-form field strength coming from an $n$-form in $(D+1)$ dimensions, an $(n-1)$-form coming from an $n$-form, and the 2-form coming from the metric. In this last case, since $\mathcal{F}$ appears for the first time, the vector $\vec{a}_{D+1}$ is zero. At the next step of the reduction, this 2-form behaves just like any other 2-form. This algorithm gives a complete construction of all the dilaton prefactors in any maximal $D$-dimensional supergravity, starting from 11-dimensional supergravity with its single 4 -form field strength. For example, in $D=10$, there is one 4 -form, one 3 -form and one 2 -form, and their (one component) dilaton vectors are given by $\left(-\frac{1}{2}\right),(1)$ and $\left(-\frac{3}{2}\right)$ respectively. In $D=9$, there is one 4 -form whose vector is $\left(-\frac{1}{2},-\frac{3}{2 \sqrt{7}}\right)$; two 3 -forms with $\left(-\frac{1}{2}, \frac{5}{2 \sqrt{7}}\right)$ and $\left(1,-\frac{1}{\sqrt{7}}\right)$; three 2 -forms with $\left(1, \frac{3}{\sqrt{7}}\right),\left(-\frac{3}{2},-\frac{1}{2 \sqrt{7}}\right)$ and $\left(0,-\frac{4}{\sqrt{7}}\right)$; and one 1 -form with $\left(-\frac{3}{2}, \frac{7}{2 \sqrt{7}}\right)$.

In general, we find that the solution to the recursion relation (2.5) is as follows. Let us denote the dilaton vectors for the 4 -form $F_{M N P Q}$, the 3 -forms $F_{M N P i}$, the 2-forms $F_{M N i j}$ and the 1-forms $F_{M i j k}$ by $\vec{a}, \vec{a}_{i}, \vec{a}_{i j}$ and $\vec{a}_{i j k}$ respectively, where $i$ labels the internal $(11-D)$ indices in $D$ dimensions. The index runs from $i=1$, corresponding to the dimension that is compactified in going from $D=11$ to $D=10$, down to $i=(11-D)$. There are also 2 -forms $\mathcal{F}_{M N}^{(i)}$ and 1 -forms $\mathcal{F}_{M i}^{(j)}$ with $i<j$, coming from the dimensional reduction of the vielbein. We denote their dilaton vectors by $\vec{b}_{i}$ and $\vec{b}_{i j}$ respectively. We find that the dilaton vectors are given by

$$
\begin{array}{rll} 
& F_{M N P Q} & \text { vielbein } \\
4-\text { form : } & \vec{a}=-\vec{g} & \\
3-\text { forms : } & \vec{a}_{i}=\vec{f}_{i}-\vec{g}, & \\
2-\text { forms : } & \vec{a}_{i j}=\vec{f}_{i}+\vec{f}_{j}-\vec{g}, & \vec{b}_{i}=-\vec{f}_{i} \\
1-\text { forms : } & \vec{a}_{i j k}=\vec{f}_{i}+\vec{f}_{j}+\vec{f}_{k}-\vec{g}, & \vec{b}_{i j}=-\vec{f}_{i}+\vec{f}_{j},
\end{array}
$$

where the vectors $\vec{g}$ and $\vec{f}_{i}$ have $(11-D)$ components in $D$ dimensions, and are given by

$$
\begin{aligned}
& \vec{g}=3\left(s_{1}, s_{2}, \ldots, s_{11-D}\right), \\
& \overrightarrow{f_{i}}=(\underbrace{0,0, \ldots, 0}_{i-1},(10-i) s_{i}, s_{i+1}, s_{i+2}, \ldots, s_{11-D}),
\end{aligned}
$$

where $s_{i}=\sqrt{2 /((10-i)(9-i))}$. It is easy to see that they satisfy

$$
\vec{g} \cdot \vec{g}=\frac{2(11-D)}{D-2}, \quad \vec{g} \cdot \vec{f}_{i}=\frac{6}{D-2}, \quad \overrightarrow{f_{i}} \cdot \vec{f}_{j}=2 \delta_{i j}+\frac{2}{D-2} .
$$


Note that the definitions in (2.6) are given for $i<j<k$, and that the vectors $\vec{a}_{i j}$ and $\vec{a}_{i j k}$ are antisymmetric in their indices. The 1 -forms $\mathcal{F}_{M i}^{(j)}$ and hence the vectors $b_{i j}$ are only defined for $i<j$, but it is sometimes convenient to regard them as being antisymmetric too, by defining $\vec{b}_{i j}=-\vec{b}_{j i}$ for $i>j$. Eqn. (2.6), together with (2.8), contains all the necessary information about the dilaton vectors in $D$-dimensional maximal supergravity.

The bosonic Lagrangian of $D=11$ supergravity is [13]

$$
\mathcal{L}=\hat{e} \hat{R}-\frac{1}{48} \hat{e} \hat{F}_{4}^{2}+\frac{1}{6} \hat{F}_{4} \wedge \hat{F}_{4} \wedge \hat{A}_{3}
$$

(We are representing the final term as an 11-form rather than a 0 -form in order to avoid writing out the $\epsilon$ tensor and all the associated indices. It is understood that the last term should be dualised.) The bosonic Lagrangian for maximal supergravity in $D$ dimensions, obtained by dimensional reduction of (2.9), is therefore

$$
\begin{aligned}
\mathcal{L}= & e R-\frac{1}{2} e(\partial \vec{\phi})^{2}-\frac{1}{48} e e^{\vec{a} \cdot \vec{\phi}} F_{4}^{2}-\frac{1}{12} e \sum_{i} e^{\vec{a}_{i} \cdot \vec{\phi}}\left(F_{3}^{i}\right)^{2}-\frac{1}{4} e \sum_{i<j} e^{\vec{a}_{i j} \cdot \vec{\phi}}\left(F_{2}^{i j}\right)^{2} \\
& -\frac{1}{4} e \sum_{i} e^{\vec{b}_{i} \cdot \vec{\phi}}\left(\mathcal{F}_{2}^{i}\right)^{2}-\frac{1}{2} e \sum_{i<j<k} e^{\vec{a}_{i j k} \cdot \vec{\phi}}\left(F_{1}^{i j k}\right)^{2}-\frac{1}{2} e \sum_{i<j} e^{\vec{b}_{i j} \cdot \vec{\phi}}\left(\mathcal{F}_{1}^{i j}\right)^{2}+\mathcal{L}_{F F A},
\end{aligned}
$$

where $F_{4}, F_{3}^{i}, F_{2}^{i j}$ and $F_{1}^{i j k}$ are the 4-form, 3-forms, 2-forms and 1-forms coming from the dimensional reduction of $\hat{F}_{4}$ in $D=11 ; \mathcal{F}_{2}^{i}$ are the 2 -forms coming from the dimensional reduction of the vielbein, and $\mathcal{F}_{1}^{i j}$ are the 1 -forms coming from the dimensional reduction of these 2 -forms. In general the field strengths appearing in the kinetic terms acquire Chern-Simons type modifications in the dimensional reduction process. In the appendix, we give the complete expressions for these modified field strengths. We denote the modified fields by untilded symbols, and the unmodifed fields, $\tilde{F}_{4}=d A_{3}$, etc., by tilded symbols. The final term $\mathcal{L}_{F F A}$ in 2.10 comes from the dimensional reduction of $\hat{F}_{4} \wedge \hat{F}_{4} \wedge \hat{A}_{3}$, and is also given in the appendix. Note that in some lower dimensions, certain higher-degree forms can be dualised to lower-degree forms. We shall always do this, so that in $D$ dimensions all forms have degree $n \leq D / 2$. The dilaton vectors of these dualised forms are equal to the negatives of the original dilaton vectors [6].

Our approach to finding $p$-brane solutions in the maximal supergravity theories is first to solve the equations following from (1.1), and then to select only those solutions that in addition satisfy the constraints implied by both $\mathcal{L}_{F F A}$ and the Chern-Simons modifications of the field strengths of the kinetic terms. First we discuss the constraints from $\mathcal{L}_{F F A}$. These arise in cases where the 0-form potentials $A_{0}^{i j k}$ appear linearly in $\mathcal{L}_{F F A}$ in a particular dimension. In order to set the $A_{0}^{i j k}$ potentials to zero consistently with their equations of motion, it is therefore necessary that the bilinear product of field strengths that occurs multiplied by $A_{0}^{i j k}$ in $\mathcal{L}_{F F A}$ should vanish. This 
imposes the following constraints:

$$
\begin{array}{ll}
D=8: & F_{4} \wedge F_{4}=0, \\
D=7: & F_{4} \wedge F_{3}^{i}=0, \\
D=6: & F_{4} \wedge F_{2}^{i j}=0, \quad F_{3}^{i} \wedge F_{3}^{j}=0, \\
D=5: & F_{3}^{[i} \wedge F_{2}^{j k]}=0, \\
D=4: & F_{2}^{[i j} \wedge F_{2}^{k l]}=0 .
\end{array}
$$

If one considers $p$-brane solutions with a purely elementary or a purely solitonic ansatz on the original fields, then the above constraints are identically satisfied. However, in $D \leq 8$ dimensions, the higher-degree field strengths can be dualised to lower-degree field strengths (or else they dualise to field strengths of the same degree). Thus the elementary (or solitonic) ansatz for a dualised field strength is equivalent to the solitonic (or elementary) ansatz for the original field strength. When the participating field strengths for a $p$-brane solution mix dualised and undualised fields, the above constraints can become non-trivial. For example, in $D=7$ we cannot have a $p$-brane solution simultaneously involving both the 3 -forms $F_{3}^{i}$ and the 3 -form $* F_{4}$ coming from the dualisation of the 4 -form. Similarly we cannot have $* F_{4}$ and $F_{2}^{i j}$ non-zero at the same time in $D=6$. On the other hand, the constraints involving both 2 -forms $F_{2}^{i j}$ and the dualised 2-forms $* F_{3}^{i}$ from 3 -forms in $D=5$ are satisified as long as each $F_{2}^{i j}$ has an index in common with each $* F_{3}^{k}$. Similar considerations apply to the 0 -brane solutions in $D=4$, where, as we may see from (2.11), if some of the 2 -forms $F_{2}^{i j}$ have elementary contributions and others have solitonic contributions, then each of the former type must have an index in common with each of the latter type.

Since the elementary and solitonic ansätze (1.4) both have the property that the field strength is closed, $d F=0$, it follows that in our $p$-brane solutions of the supergravity theories, we must require that all the Chern-Simons modifications to the field strengths should preserve this property. In addition, field strengths of degrees other than the degree of the fields participating in the solution must be zero. It therefore follows that the exterior derivatives of all the Chern-Simons modifications must vanish. This gives rise to non-trivial constraints in $D=5$ and $D=4$. For example, consider 0 -brane (particle) solutions using 2-forms in $D=4$. Since the forms of all other degrees are assumed to be zero, this means in particular that the 0 -form potentials $\mathcal{A}_{0}^{i j}$ are zero, and hence $\gamma^{i j}=\delta^{i j}$ in (A.6). It follows that the vanishing of the 3 -forms $F_{3}^{i}$ gives rise to the non-trivial constraint

$$
F_{2}^{i j} \wedge \mathcal{F}_{2}^{j}=0
$$

For the purely elementary or purely solitonic ansatz, this constraint is identically satisfied. However, when the solution has mixed elementary and solitonic contributions, the constraint vanishes only 
when the corresponding field strengths do not have a common index.

In this paper, our principal interest is in the $p$-brane solutions with either a pure elementary or a pure solitonic ansatz for each field strength. In certain dimensions, where a field strength dualises to one of the same degree, we can also in principle consider $p$-brane solutions in which the canonical field strength has both elementary and solitonic contributions. These dyonic configurations may or may not satisfy the constraints implied by the $\mathcal{L}_{F F A}$ term and the Chern-Simons modifications to the field strengths. For example, we can do this for the 3 -form field strengths in $D=6$ and for the 2-form field strengths in $D=4$. The situation is different in $D=8$, where such a mixed elementary and solitonic solution does not exist for the 4-form field strength, as can be easily seen from the above constraint. (However, the situation is different if a non-zero $A_{0}^{i j k}$ is allowed; in fact such a solution does exist [14], but it lies outside the class of solutions that we are considering in this paper. In all the solutions we are considering, there is no non-zero contribution from $\mathcal{L}_{F F A}$.) Finally, we remark that when one considers $p$-brane solutions for 1-form field strengths in $D=5$, the equations of motion for the 0 -form potentials $A_{0}^{i j k}$ are modified by an $F_{4} \wedge F_{1}^{i j k}$ term. This additional term vanishes if we only consider $p$-brane solutions that do not involve the dualised 1 -form $* F_{4}$.

\section{General solutions and Bogomol'nyi matrices}

We have seen in the introduction that the metric of a $p$-brane solution is specified once the $\Delta$ of the dilaton prefactor of the field strength is given. We have stated also that the values of $\Delta$ for all the original field strengths that are obtained simply by Kaluza-Klein dimensional reduction are given by $\Delta=4$. (It is very easy to verify this by substituting $\vec{a} \cdot \vec{a}$ into eqn. (1.3), using the expression for the $\vec{a}$ 's that we obtained in the previous section.) Thus if we use any one of these field strengths to construct a $p$-brane solution while setting all the other field strengths to zero, it will have $\Delta=4$. However, as we shall now show, there are other solutions in which a linear combination of field strengths of a given degree, and a corresponding linear combination of the dilatons, are involved. These solutions will in general have $\Delta<4$. The possibility of making such linear combinations depends crucially on the properties of the dot products of the dilaton vectors for the various field strengths. Let us consider a bosonic Lagrangian with $N$-forms $F_{\alpha}$, with $\alpha=1,2, \ldots N$, namely

$$
\mathcal{L}=e R-\frac{1}{2} e(\partial \vec{\phi})^{2}-\frac{1}{2 n !} \sum_{\alpha=1}^{N} e^{\vec{a}_{\alpha} \cdot \vec{\phi}} F_{\alpha}^{2}
$$


To find a solution involving all these field strengths and a single linear combination $\phi$ of dilaton fields, we perform a rotation in the space of dilatons by writing

$$
\vec{\phi}=\vec{n} \phi+\vec{\phi}_{\perp} \quad \text { such that } \quad \vec{a}_{\alpha} \cdot \vec{n}=a \quad \text { for all } \quad \alpha
$$

where $\vec{n}$ is a unit vector and $\vec{n} \cdot \vec{\phi}_{\perp}=0$. Note that the requirement that all the $\vec{a}_{\alpha}$ have the same projection on $\vec{n}$ is necessary so that the participating field strengths have a Lagrangian of the form (1.1), with a common exponential prefactor. In order to be able to set $\vec{\phi}_{\perp}$ to zero consistently with its equations of motion, it is therefore necessary that

$$
\sum_{\alpha} \vec{a}_{\alpha} F_{\alpha}^{2}=a \vec{n} \sum_{\alpha} F_{\alpha}^{2}
$$

Taking the dot product with $\vec{a}_{\beta}$, we obtain

$$
\sum_{\alpha} A_{\alpha \beta} F_{\alpha}^{2}=a^{2} \sum_{\alpha} F_{\alpha}^{2}
$$

where $A_{\alpha \beta} \equiv \vec{a}_{\alpha} \cdot \vec{a}_{\beta}$. If the matrix $A_{\alpha \beta}$ is invertible, we therefore have

$$
\begin{aligned}
F_{\beta}^{2} & =a^{2} \sum_{\alpha}\left(A^{-1}\right)_{\alpha \beta} \sum_{\gamma} F_{\gamma}^{2} \\
a^{2} & =\left(\sum_{\alpha, \beta}\left(A^{-1}\right)_{\alpha \beta}\right)^{-1}
\end{aligned}
$$

which gives rise to a $p$-brane solution with $\Delta=\left(\sum_{\alpha, \beta}\left(A^{-1}\right)_{\alpha \beta}\right)^{-1}+2 d \tilde{d} /(D-2)$. In this case, we can easily see that $a \neq 0$, and hence the unit vector $\vec{n}$ can be found from eqn (3.3).

When the matrix $A_{\alpha \beta}$ is singular, the analysis is different. Unlike the the previous case, we can have solutions to the homogeneous equation (3.4) with more than just the one rescaling parameter. We shall first consider solutions with only the rescaling parameter. In this case, the solutions must have $a=0$ since the matrix $A_{\alpha \beta}$ is singular and has a zero eigenvector, which solves eqn. (3.4). This corresponds to $\Delta=2 d \tilde{d} /(D-2)$. When a solution to eqn. (3.4) has extra free parameters in addition to the overall scale, we can have values of $a$ other than zero. However, the values that occur are still discrete, and independent of the values of the parameters. Thus these values of $a$ are just repetitions of those that occurred for cases with fewer than $N$ participating field strengths, since we may without loss of generality choose the parameters such that one or more of the field strengths vanishes, thereby reducing the number of participating fields to a previously considered case. For the purpose of this paper, therefore, we need only consider the cases where $A_{\alpha \beta}$ is nonsingular, or, if it is singular, it should have only one zero eigenvalue, leading to $a=0$. A particular class of singular $A_{\alpha \beta}$ 's are the ones corresponding to the cases when $N$ is larger than the number of dilatonic scalar fields. In this case, we find that all the solutions involve more than the one overall 
scale parameter, and thus need not be considered further. Thus in dimension $D$, we need consider at most $(11-D)$ non-vanishing field strengths. Note that equations (3.3) and (3.4) determine the relations between the squares of the field strengths, rather than the field strengths themselves. The remaining freedom to choose the signs of the corresponding Page charges sometimes affects the supersymmetry properties of the solutions, as we shall see later.

In the next three sections, we shall carry out the search for solutions in all dimensions $D \geq 4$, for all $n$-forms with $n \leq 4$. For each solution, we shall also examine its supersymmetry. In order to determine how much supersymmetry is preserved by the solutions, we now develop a general formalism using the Bogomol'nyi matrix, i.e. the commutator of conserved supercharges. For each component of unbroken supersymmetry, this matrix will have a zero eigenvalue. We can construct the Bogomol'nyi matrices by first constructing the one for 11-dimensional supergravity, and then obtaining those for $D<11$ by dimensional reduction.

Given a spinor $\epsilon$ that is asymptotically constant as $r \rightarrow \infty$, the associated supercharge per unit spatial $p$-volume of the the $p$-brane is given by [1]

$$
Q_{\epsilon}=\int_{\partial \Sigma} \bar{\epsilon} \Gamma^{A B C} \psi_{C} d \Sigma_{A B}
$$

where $\partial \Sigma$ is the $(\tilde{d}+1)$-sphere with radius $r$ in the transverse space. The commutator of the resulting supercharges is given by

$$
\left[Q_{\epsilon_{1}}, Q_{\epsilon_{2}}\right]=\delta_{\epsilon_{1}} Q_{\epsilon_{2}}=\int_{\partial \Sigma} N^{A B} d \Sigma_{A B}
$$

where $N^{A B}=\bar{\epsilon}_{1} \Gamma^{A B C} \delta_{\epsilon_{2}} \psi_{C}$. From the transformation rule for the gravitino in $D=11$ supergravity, we therefore obtain the Nester form

$$
N^{A B}=\bar{\epsilon}_{1} \Gamma^{A B C} D_{C} \epsilon_{2}+\frac{1}{8} \bar{\epsilon}_{1} \Gamma^{C_{1} C_{2}} \epsilon_{2} F^{A B}{ }_{C_{1} C_{2}}+\frac{1}{96} \bar{\epsilon}_{1} \Gamma^{A B C_{1} \ldots C_{4}} \epsilon_{2} F_{C_{1} \ldots C_{4}}
$$

Since only the $d \Sigma_{0 r}$ component of the $p$-brane spatial volume element contributes, we may read off the Bogomol'nyi matrix $\mathcal{M}$ from the integral

$$
\frac{1}{\omega_{\tilde{d}+1}} \int_{\partial \Sigma \text { at } r \rightarrow \infty} N^{0 r} r^{\tilde{d}+1} d \Omega_{(\tilde{d}+1)}=\epsilon_{1}^{\dagger} \mathcal{M} \epsilon_{2},
$$

where $\omega_{\tilde{d}+1}$ is the volume of the unit $(\tilde{d}+1)$-sphere. If there is an unbroken supersymmetry, then there exists a Killing spinor such that eqn. (3.7) vanishes. In other words, the Bogomol'nyi matrix (3.9) has a zero eigenvalue for each component of the unbroken supersymmetry.

We can now use the Bogomol'nyi matrix to study the supersymmetry of the $p$-brane solutions in $D=11$ dimensions. There is only one field strength in $D=11$ supergravity, namely the 4 -form, which gives rise to an elementary membrane and a solitonic 5-brane whose metrics are given by 
(1.5). Note that for the elementary ansatz for field strengths given by (1.4), the last term in (3.8) vanishes, whilst the second term vanishes for the solitonic ansatz. Substituting the solutions into eqn. (3.9), we obtain

$$
\begin{aligned}
\text { elementary : } & \mathcal{M}=m \mathbb{1}+u \Gamma_{012}, \\
\text { solitonic : } & \mathcal{M}=m \mathbb{1}+v \Gamma_{\hat{1} \hat{2} \hat{3} \hat{4} \hat{5}},
\end{aligned}
$$

where the hats indicate index values in the transverse space, while indices without hats live in the world-brane volume. The parameter $m$ denotes the mass per unit volume of the $p$-brane, and $u=\frac{1}{4 \omega_{7}} \int_{S^{7}} * F$ and $v=\frac{1}{4 \omega_{4}} \int_{S^{4}} F$ are precisely the electric and magnetic Page charges of the field strength [15]. They are given by

$$
m=\frac{\lambda}{4}, \quad u=\frac{\lambda}{4}=v .
$$

The eigenvalues of the Bogomol'nyi matrix (3.10) can be easily obtained without needing to decompose the 11-dimensional $\Gamma$ matrices, by invoking the Hamilton-Cayley theorem that a matrix satisfies its own characteristic equation. For example, let us consider the elementary case:

$$
(\mathcal{M}-m \mathbb{1})^{2}=\left(u \Gamma_{012}\right)^{2}=u^{2} \quad \Rightarrow \quad \mu=(m \pm u),
$$

where $\mu$ denotes the eigenvalues of $\mathcal{M}$. Since $\Delta=4$ in $D=11$, it is easy to see that $m=u$, and so the $32 \times 32$ matrix $\mathcal{M}$ has 16 zero eigenvalues, for both the elementary membrane and solitonic 5 -branes solutions. We shall write the eigenvalues as $2 m\left(0_{16}, 1_{16}\right)$, where the subscripts denote the degeneracies. Thus both the elementary and solitonic solutions break half the supersymmetry and their mass/charge ratio is 1. Although the Hamilton-Cayley theorem provides a simple way to calculate the eigenvalues, it is not always easy to determine the degeneracies by this approach. Knowing the degeneracies is of particular interest for the supersymmetric $p$-brane solutions, since it determines the fraction of the $D=11$ supersymmetry that is preserved. For the supersymmetric cases, we calculate the full set of 32 eigenvalues, using an explicit representation of $32 \times 32$ gamma matrices in $D=11$.

The above analysis of supersymmetry can easily be generalised to lower dimensions. In fact the Nester form for maximal supergravity in any dimension is just the Kaluza-Klein dimensional reduction of the 11-dimensional expression (3.8). For example, it follows from eqns (2.1), (2.2) and (3.8) that the Nester form for type IIA supergravity in $D=10$ is given by

$$
\begin{aligned}
N^{A B}= & \bar{\epsilon}_{1} \Gamma^{A B C} D_{C} \epsilon_{2}+e^{-\frac{3}{4} \phi} \bar{\epsilon}_{1} \Gamma_{10}\left(\frac{1}{4} \mathcal{F}^{A B}+\frac{1}{8} \Gamma^{A B C D} \mathcal{F}_{C D}\right) \epsilon_{2} \\
& +e^{\frac{1}{2} \phi} \bar{\epsilon}_{1} \Gamma_{10}\left(-\frac{1}{4} \Gamma^{C} F_{C}^{A B}-\frac{1}{24} \Gamma^{A B C D E} F_{C D E}\right) \epsilon_{2} \\
& +e^{-\frac{1}{4} \phi} \bar{\epsilon}_{1}\left(\frac{1}{8} \Gamma^{C D} F_{C D}^{A B}+\frac{1}{96} \Gamma^{A B C D E F} F_{C D E F}\right) \epsilon_{2}
\end{aligned}
$$


The Nester form becomes increasingly complicated as we descend through the dimensions, since more and more antisymmetric tensors are generated. However, for the purpose of studying the supersymmetries of $p$-brane solutions, some simplifications can be made. First, note that the dilaton factor for each field strength is precisely the square root of the dilaton factor for the kinetic term of the same field strength that appears in the Lagrangian. In fact it follows from (1.5) that all these dilaton factors can be set to unity since the Bogomol'nyi matrix we are considering is defined at $r=\infty$. As we showed above, in order to obtain the eigenvalues of a Bogomol'nyi matrix, we do not need to decompose the $\Gamma$ matrices into world-volume and transverse space factors. Furthermore, we do not need to decompose the 11-dimensional $\Gamma$ matrices into the product of $D$-dimensional spacetime and compactified $(11-D)$-dimensional factors. This greatly simplifies the discussion for lower dimensions.

In order to present the general Bogomol'nyi matrix for arbitrary forms and arbitrary dimensions, we first establish a notation for the Page charges of the various field strengths:

$\begin{array}{rcccccc} & F_{4} & F_{3}^{i} & F_{2}^{i j} & F_{1}^{i j k} & \mathcal{F}_{2}^{i} & \mathcal{F}_{1}^{i j} \\ \text { elementary : } & u & u_{i} & u_{i j} & & p_{i} & \\ \text { solitonic : } & v & v_{i} & v_{i j} & v_{i j k} & q_{i} & q_{i j},\end{array}$

where the elementary Page charges are given by $\frac{1}{\omega_{D-n}} \int_{S^{(D-n)}} * F_{n}$ and the solitonic Page charges are given by $\frac{1}{4 \omega_{n}} \int_{S^{n}} F_{n}$. Note that there are no elementary $p$-brane solutions for the 1-form field strengths. We find that the general Bogomol'nyi matrix in $D$ dimensions is given by

$$
\begin{aligned}
\mathcal{M}= & m \mathbb{1}+u \Gamma_{012}+u_{i} \Gamma_{01 i}+\frac{1}{2} u_{i j} \Gamma_{0 i j}+p_{i} \Gamma_{0 i} \\
& +v \Gamma_{\hat{1} \hat{2} \hat{3} \hat{4} \hat{5}}+v_{i} \Gamma_{\hat{1} \hat{2} \hat{3} \hat{4} i}+\frac{1}{2} v_{i j} \Gamma_{\hat{1} \hat{2} \hat{3} i j}+\frac{1}{6} v_{i j k} \Gamma_{\hat{1} \hat{2} i j k}+q_{i} \Gamma_{\hat{1} \hat{2} \hat{3} i}+\frac{1}{2} q_{i j} \Gamma_{\hat{1} \hat{2} i j},
\end{aligned}
$$

where the first line contains the contributions for elementary solutions, and the second line contains the contributions for solitonic solutions. For a given degree $n$ of antisymmetric tensor field strength, only the terms with the corresponding Page charges, as indicated in (3.14), will occur. As always, the indices $0,1, \ldots$ run over the dimension of the $p$-brane worldvolume, $\hat{1}, \hat{2}, \ldots$ run over the transverse space of the $y^{m}$ coordinates, and $i, j, \ldots$ run over the dimensions that were compactified in the Kaluza-Klein reduction from 11 to $D$ dimensions. The mass per unit $p$-volume $m$ in (3.15) arises from the connection term in the covariant derivative in the Nester form, and it is given by $m=\frac{1}{2} \lim _{r \rightarrow \infty}\left(B^{\prime}-A^{\prime}\right) e^{-B} r^{\tilde{d}+1}$. Thus the masses and Page charges of the solutions of (1.1) that we discussed in section 1 are given for all $D$ and $d$ by 


\begin{tabular}{|c|c|c|c|c|}
\hline & Elementary & Solitonic & Dyonic, $a^{2}=n-1$ & Dyonic, $a^{2}=0$ \\
\hline \hline Mass $m$ & $\frac{1}{2 \sqrt{\Delta}} \lambda$ & $\frac{1}{2 \sqrt{\Delta}} \lambda$ & $\frac{1}{2 \sqrt{\Delta}}\left(\lambda_{1}+\lambda_{2}\right)$ & $\frac{1}{2 \sqrt{\Delta}} \sqrt{\lambda_{1}^{2}+\lambda_{2}^{2}}$ \\
\hline Charge $P_{\text {ele }}$ & $\frac{1}{4} \lambda$ & 0 & $\frac{1}{4} \lambda_{1}$ & $\frac{1}{4} \lambda_{1}$ \\
\hline Charge $P_{\text {sol }}$ & 0 & $\frac{1}{4} \lambda$ & $\frac{1}{4} \lambda_{2}$ & $\frac{1}{4} \lambda_{2}$ \\
\hline
\end{tabular}

Table 1: Mass and Page charges for $p$-brane solutions

As one can see from (3.12), the existence of zero eigenvalues of a Bogomol'nyi matrix depends on two factors: the $\Gamma$ matrix structure and the $\Delta$ value of the solution. Both of these two factors are governed by the field strengths that participate in the solution. In the next three sections, we shall exhaustively search for $p$-brane solutions using $n$-forms with $n=4,3,2,1$ in all dimensions $11 \geq D \geq 4$. In the case of 4 -forms and 3 -forms we descend only to $D=8$ and $D=6$ respectively, since below these dimensions, the forms will be dualised to forms of lower degree. It will be understood in everything that follows that we are always discussing $p$-brane solutions in the maximal supergravity that is obtained from $D=11$ by dimensional reduction.

\section{$4 \quad p$-branes for 4-form and 3-form field strengths}

\subsection{4-form field strengths}

We first discuss $p$-brane solutions for 4 -form field strengths in $D \geq 8$ dimensions. There is only one 4-form in each dimension $D$. Therefore, in this case, there is no possibility of truncation of the dilatonic scalar fields, and so there is a unique solution, with $\Delta=4$. When it is elementary, it is a membrane; when it is solitonic, it is a $(D-6)$-brane. The Bogomol'nyi matrices for all these dimensions take the same form (3.10) as in 11 dimensions. Thus the eigenvalues are given by $m \pm u$ for the elementary cases and $m \pm v$ for the solitonic cases. It follows from eqn. (3.11) that all these solutions preserve half of the 11-dimensional supersymmetry. Note that in $D=8$, one could consider dyonic membrane solutions given by (1.9). However, as we discussed in section 2 , such a solution is ruled out by the constraint given in 2.11). (It is worth remarking that one can nevertheless construct a more general kind of dyonic solution where a 0-form potential is in addition involved [14].)

In fact the above discussion applies also to the $p$-brane solutions for $n$-forms of any other degree, if only one of the original field strengths participates in the solution. In other words, any $p$-brane solution with $\Delta=4$ preserves half of the 11-dimensional supersymmetry, regardless of the dimension $D$ and the degree $n$ of the form. Thus in the following discussion, we shall consider only the more complicated cases where more than one field strength is involved, and hence $\Delta<4$. 


\section{$4.2 \quad 3$-form field strengths}

There are $(11-D) 3$-forms in $D \geq 6$ dimensions, except in $D=7$ where there is an extra 3 -form coming from the dualisation of the 4 -form. The 3 -forms are obtained from dimensional reduction of the 4 -form in $D=11$. Leaving aside the extra 3 -form in seven dimensions, they can be labelled by $F^{A B C i}$, where $i$ runs over the $(11-D)$ compactified dimensions. In the case of $D=7$, it turns out that the extra 3-form can be appended to these by extending the range of the index $i$. The dilaton vectors $\vec{a}_{i}$ for the corresponding 3 -form field strengths $F^{A B C i}$, which are given by (2.6), turn out to have the following dot products

$$
\vec{a}_{i} \cdot \vec{a}_{j}=2 \delta_{i j}-\frac{2(D-6)}{D-2}
$$

Let us now suppose that there are $N 3$-forms participating in a $p$-brane solution, where $N$ is less than or equal to the number of dilatonic scalar fields, i.e. $N \leq(11-D)$. It follows from the first equation of (3.5) that all these 3-forms are equal, i.e. $F_{\alpha}^{2}=F^{2} / N$ for all $\alpha$, where $F$ is the canonically normalised field strength that appears in the Lagrangian (1.1) after setting the non-participating fields to zero. Thus it follows from eqn. (3.3) that

$$
a^{2}=\left(\frac{1}{N} \sum_{\alpha} \vec{a}_{\alpha}\right)^{2}=\frac{2}{N}-\frac{2(D-6)}{D-2},
$$

which implies that

$$
\Delta=2+\frac{2}{N}=4,3, \frac{8}{3}, \frac{5}{2}, \frac{12}{5} .
$$

This implies that there are $(11-D)$ inequivalent $p$-brane solutions using 3 -form field strengths in $D$ dimensions. The elementary solutions are strings, while the solitonic solutions are $(D-5)$-branes.

In order to study the supersymmetry of these $p$-brane solutions we consider their Bogomol'nyi matrices, given in (3.15). In $D=10,9,8$, we therefore have

$$
\begin{aligned}
\text { elementary: } & \mathcal{M}=m \mathbb{1}+u_{i} \Gamma_{01 i}, \quad \text { eigenvalues : } \quad \mu=m \pm \sqrt{u_{i} u_{i}}, \\
\text { solitonic: } & \mathcal{M}=m \mathbb{1}+v_{i} \Gamma_{\hat{1} \hat{2} \hat{3} \hat{4} i}, \quad \text { eigenvalues : } \quad \mu=m \pm \sqrt{v_{i} v_{i}},
\end{aligned}
$$

where we denote the elementary and solitonic Page charges by $u_{i}$ and $v_{i}$ respectively, and the index $i$ runs over the compactified dimensions. As we discussed above, for a $p$-brane solution involving $N \leq(11-D)$ field strengths, we have $F_{\alpha}^{2}=F^{2} / N$ for all $\alpha$, and hence $u_{\alpha}=u / \sqrt{N}$ or $v_{\alpha}=v / \sqrt{N}$ where $u$ and $v$ are the Page charges for the canonically normalised field strength $F$, i.e. $u=\frac{1}{4} \lambda=v$. Thus the eigenvalues for both the elementary and solitonic solutions are given by $\mu=\frac{1}{4} \lambda\left(\frac{2}{\sqrt{\Delta}} \pm 1\right)$, and can be zero only when $\Delta=4$, in which case half of the supersymmetry is preserved. 
In $D=7$, there is an extra 3 -form coming from the dualisation of the 4 -form. The Bogomol'nyi matrices are given by

$$
\begin{array}{rlll}
\text { elementary : } & \mathcal{M}=m \mathbb{1}+u_{i} \Gamma_{01 i}+u \Gamma_{\hat{1} \hat{2} \hat{3} \hat{4} \hat{5}}, & \text { eigenvalues : } & \mu=m \pm \sqrt{u_{i} u_{i}+u^{2}} \\
\text { solitonic : } & \mathcal{M}=m \mathbb{1}+v_{i} \Gamma_{\hat{1} \hat{2} \hat{3} \hat{4} i}+v \Gamma_{012}, \quad \text { eigenvalues: } & \mu=m \pm \sqrt{v_{i} v_{i}+v^{2}}
\end{array}
$$

where $u$ and $v$ denote the elementary and solitonic Page charges of the dual of the 4-form field strength. Note that, as we discussed for the solutions, $u$ and $v$ play an equivalent rôle to $u_{i}$ and $v_{i}$ respectively in the Bogomol'nyi matrices, and therefore we can extend the range of the index $i$ by 1 . Thus as in the cases of $D \geq 8$, the eigenvalues are given by $\mu=\frac{1}{4} \lambda\left(\frac{2}{\sqrt{\Delta}} \pm 1\right)$. Again the supersymmetry is completely broken unless $N=1$ and hence $\Delta=4$, in which case half of the supersymmetry is preserved.

In $D=6$, the Bogomol'nyi matrices are given by

$$
\begin{aligned}
\qquad \mathcal{M}= & m \mathbb{1}+u_{i} \Gamma_{01 i}+v_{i} \Gamma_{\hat{1} \hat{2} \hat{3} \hat{4} i}, \\
\text { eigenvalues : } & \mu=m \pm \sqrt{\left(u_{i} \pm v_{i}\right)^{2}}
\end{aligned}
$$

(Note that owing to the more complex $\Gamma$-matrix structure, the characteristic equation for $\mathcal{M}$ is quartic here rather than quadratic, giving rise to the two independent \pm signs.) The elementary and solitonic solutions correspond to $v_{i}=0$ and $u_{i}=0$ respectively. For these types of solutions, the supersymmetry properties are identical to the case when $D \geq 7$, namely all solutions break all the supersymmetry except when $\Delta=4$, in which case they preserve half the supersymmetry.

In $D=6$, since the dimension is twice the degree of the 3-form field strengths, we can also construct dyonic string solitons, where the field strengths involve both elementary and solitonic contributions. There could in principle be two different types of dyonic solution. In the first type, some field strengths would be purely elementary (with electric charge) while others would be purely solitonic (with magnetic charge). However, such configurations do not satisfy the constraint given in (2.11). In the second type of solution, the canonical field strength has both electric and magnetic charges, and hence all the participating field strengths have the same ratio of the two charges. This configuration does satisfy the constraint in (2.11). Thus we can have such dyonic solutions with $N=1,2,3,4$ and 5 field strengths, and the correponding values of $\Delta$ are given by (4.3). In general, the equations of motion reduce to the two second-order differential equations (1.7). When $N=1$, and hence $\Delta=4$, we have a simple solution given by (1.9). The mass per unit length of this dyonic string is $m=\frac{1}{4}\left(\lambda_{1}+\lambda_{2}\right)$, and the Page charges are $u=\frac{1}{4} \lambda_{1}$ and $v=\frac{1}{4} \lambda_{2}$. The eigenvalues of the Bogomol'nyi matrix are given by

$$
\mu=m \pm u \pm v=\left\{0_{8},\left(\frac{1}{2} \lambda_{1}\right)_{8},\left(\frac{1}{2} \lambda_{2}\right)_{8},\left(\frac{1}{2}\left(\lambda_{1}+\lambda_{2}\right)\right)_{8}\right\}
$$


where, as usual, the subscripts on the eigenvalues indicate their degeneracies. Thus the solution preserves $\frac{1}{4}$ of the supersymmetry [12]. When either $\lambda_{1}=0$ or $\lambda_{2}=0$, the solution reduces precisely to the previously-discussed purely solitonic and purely elementary solutions, which preserve $\frac{1}{2}$ of the supersymmetry. When $\lambda_{1}=\lambda_{2}$, in which case the field strength becomes self-dual and the dilaton vanishes, the solution is in fact precisely equivalent to the self-dual string in $D=6$ self-dual supergravity, which we shall discuss below. When $\lambda_{1}=-\lambda_{2}$, the field strength is anti-self-dual, and we have a massless string which preserves $\frac{1}{2}$ of the supersymmetry; however, the eigenvalues, given by (4.7), for such a solution are not positive semi-definite. In this case, the dilaton field does not vanish, and hence the solution is distinct from the anti-self-dual string in $D=6$ anti-self-dual gravity. It is worth remarking that the eigenvalues (4.7) for these dyonic solutions of the second type are quite different from those for all the solutions we have discussed previously. In those cases, the eigenvalues are non-negative as long as the mass per unit $p$-volume is positive. However, for the dyonic solutions of the second type, one can easily see that the eigenvalues (4.7) of the Bogomol'nyi matrices can take both signs, even when the mass is positive.

In the above discussion, we saw that the field strength of the solution could be chosen to be either self-dual or anti-self-dual. In fact, one can alternatively impose a self-dual (or anti-self-dual) condition on a 3-form field strength so as to truncate the supergravity theory itself to an (anti)self-dual theory [16]. In this case, the dilatonic fields are all consistently truncated from the theory. This gives rise to a self-dual (or anti-self-dual) string soliton with $\Delta=2$. The metric is given by (1.10) with $\lambda_{1}=\lambda_{2}=\lambda$. The mass per unit length is given by $m=\frac{1}{2} \sqrt{\left(\lambda_{1}^{2}+\lambda_{2}^{2}\right) / \Delta}=\frac{1}{2} \lambda$; the Page charges of the solution comprise an electric charge $u$ and a magnetic charge $v$, with $u=v=\frac{1}{4} \lambda$ $\left(v=-\frac{1}{4} \lambda\right.$ for the anti-self-dual case). The eigenvalues of the Bogomol'nyi matrix are given by $\mu=\frac{\lambda}{4}(2 \pm 1 \pm 1)=m\left\{0_{8}, 1_{16}, 2_{8}\right\}$, and so a quarter of the $D=11$ supersymmetry is preserved in this (anti)-self-dual case. Note that the mass per unit $p$-volume of the self-dual (anti-self-dual) solution in the previous paragraph is given by $m=u+v$, whilst the mass of these solution in $D=6$ self-dual (anti-self-dual) supergravity is given by $m=\sqrt{u^{2}+v^{2}}$.

\section{$5 \quad p$-branes for 2-form field strengths}

The structure of the 2-form field strengths is much complicated than that for 4 -forms and 3-forms. There are two sources of 2-form fields strengths, namely the vielbein and the dimensional reduction of the 4 -form in $D=11$. Furthermore, in $D=6$, there is an additional 2-form coming from the dualisation of the 4 -form, and in $D=5$, there are six additional 2 -forms coming from the dualisation of the 3 -forms. Thus we shall discuss $D \geq 7, D=6, D=5$ and $D=4$ separately. The 
elementary solutions are particles, while the solitonic solutions are $(D-4)$-branes.

\section{1 $D \geq 7$}

When $D \geq 7$, there are $(11-D)$ 2-forms $\mathcal{F}_{M N}^{(i)}$ coming from the vielbein, and $\frac{1}{2}(11-D)(10-D) 2$ forms $F_{M N i j}$ coming from the 4-form field strength, where $i$ runs over the compactified dimensions. We are denoting the dilaton vectors by $\vec{b}_{i}$ and $\vec{a}_{i j}$ respectively; they are given in eqn. (2.6). It follows from eqn. (2.8) that they satisfy

$$
\begin{aligned}
\vec{b}_{i} \cdot \vec{b}_{j} & =2 \delta_{i j}+\frac{2}{D-2} \\
\vec{b}_{i} \cdot \vec{a}_{j k} & =-2 \delta_{i j}-2 \delta_{i k}+\frac{2}{D-2}, \quad j<k, \\
\vec{a}_{i j} \cdot \vec{a}_{k l} & =2 \delta_{i k}+2 \delta_{j l}+2 \delta_{i l}+2 \delta_{j k}-\frac{2(D-3)}{D-2}, \quad i<j, k<l .
\end{aligned}
$$

The Bogomol'nyi matrix for the elementary case is $\mathcal{M}=m \mathbb{1}+p_{i} \Gamma_{0 i}+\frac{1}{2} u_{i j} \Gamma_{0 i j}$. Its characteristic equation is quartic, and the eigenvalues are

$$
\begin{array}{ll}
\mu=m \pm \sqrt{p_{i} p_{i}+\frac{1}{2} u_{i j} u_{i j} \pm 2 \sqrt{u_{i j} p_{i} u_{k j} p_{k}}}, & D=10,9,8 \\
\mu=m \pm \sqrt{p_{i} p_{i}+\frac{1}{2} u_{i j} u_{i j} \pm \sqrt{\frac{1}{16}\left(\epsilon_{i j k l} u_{i j} u_{k l}\right)^{2}+4 u_{i j} p_{i} u_{k j} p_{k}}}, \quad D=7 .
\end{array}
$$

For the solitonic case, the analysis is analogous, and the eigenvalues are given by (5.2) with the elementary Page charges $p_{i}$ and $u_{i j}$ replaced by the solitonic Page charges $q_{i}$ and $v_{i j}$.

Using eqns (5.1) and (5.2), we can obtain all the $p$-brane solutions for 2-form field strengths in $D \geq 7$, and study their supersymmetry properties. As we discussed previously, the $p$-brane solutions involving only one original 2-form have $\Delta=4$, and preserve half of the supersymmetry. For $N=2$ participating 2-forms, which can happen in $D \leq 9$, there are two inequivalent solutions for each $D$, with $\Delta=3$ and $\Delta=2$. For each value of $\Delta$, there may be more than one choice of field strengths to achieve it; however, as long as the ratio of field strengths is the same for the different choices, we expect that they give rise to identical eigenvalues in the Bogomol'nyi matrix, and hence the same supersymmetry property. Thus we shall present only one example for each inequivalent solution:

$$
\begin{array}{lll}
\Delta=3, & p_{1}=p_{2}=\frac{\lambda}{4 \sqrt{2}}, & \mu=\frac{\lambda}{4}\left(\frac{2}{\sqrt{3}} \pm 1\right), \\
\Delta=2, & p_{1}=u_{12}=\frac{\lambda}{4 \sqrt{2}}, & \mu=\frac{\lambda}{4 \sqrt{2}}(2 \pm 1 \pm 1) .
\end{array}
$$

We see that the $p$-brane solutions with $\Delta=3$ break all the supersymmetry, and the $p$-brane solutions with $\Delta=2$ preserve a quarter of the supersymmetry, with the eigenvalues given by $m\left\{0_{8}, 1_{16}, 2_{8}\right\}$. 
For $N=3$ field strengths, which can happen in $D \leq 8$, there are also two inequivalent solutions for each $D$, namely

$$
\begin{array}{ll}
\Delta=\frac{8}{3}, & \left\{p_{1}, p_{2}, p_{3}\right\}=\frac{\lambda}{4 \sqrt{3}}\{1,1,1\}, \quad \mu=\frac{\lambda}{4}\left(\sqrt{\frac{3}{2}} \pm 1\right) \\
\Delta=\frac{12}{7}, & \left\{p_{1}, p_{2}, u_{12}\right\}=\frac{\lambda}{4 \sqrt{7}}\{\sqrt{2}, \sqrt{2}, \sqrt{3}\}, \quad \mu=\frac{\lambda}{4}\left(\sqrt{\frac{7}{3}} \pm \sqrt{1 \pm \frac{4 \sqrt{3}}{7}}\right) .
\end{array}
$$

These solutions break all the supersymmetry.

For $N=4$ field strengths, which can happen in $D \leq 7$, there are 3 inequivalent solutions, given by

$$
\begin{aligned}
& \Delta=\frac{5}{2}, \quad\left\{p_{1}, p_{2}, p_{3}, p_{4}\right\}=\frac{\lambda}{8}\{1,1,1,1\}, \quad \mu=\frac{\lambda}{4}\left(\sqrt{\frac{8}{5}} \pm 1\right) \\
& \Delta=\frac{5}{3}, \quad\left\{p_{1}, p_{2}, u_{12}, u_{13}\right\}=\frac{\lambda}{4 \sqrt{6}}\{\sqrt{2}, 1, \sqrt{2}, 1\}, \quad \mu=\frac{\lambda}{4}\left(\sqrt{\frac{12}{5}} \pm \sqrt{1 \pm \frac{\sqrt{8}}{3}}\right) \\
& \Delta=\frac{8}{5}, \quad\left\{p_{1}, u_{12}, u_{13}, u_{14}\right\}=\frac{\lambda}{4 \sqrt{5}}\{\sqrt{2}, 1,1,1\}, \quad \mu=\frac{\lambda}{4}\left(\sqrt{\frac{5}{2}} \pm \sqrt{1 \pm \frac{2 \sqrt{6}}{5}}\right) .
\end{aligned}
$$

All these solutions, which break all the supersymmetry, satisfy the constraints implied by both $\mathcal{L}_{F F A}$ and the Chern-Simons modifications that we discussed in section 2. Note that in order to get $\Delta=\frac{5}{3}$, we can also have $\left\{p_{1}, p_{2}, p_{3}, u_{12}\right\}=\frac{\lambda}{4 \sqrt{6}}\{\sqrt{2}, \sqrt{2}, \sqrt{-1}, \sqrt{3}\}$. The eigenvalues are given by $\mu=\frac{\lambda}{4}\left(\sqrt{\frac{12}{5}} \pm \sqrt{1 \pm \frac{2}{\sqrt{3}}}\right)$. In this case, one of the field strengths is imaginary and $\mu$ includes complex values. The solution is unphysical. From now on, we shall discard these types of solutions.

\section{$5.2 D=6$}

In $D=6$, there are five 2-forms $\mathcal{F}_{M N}^{(i)}$ coming from the vielbein, ten $F_{M N i j}$ coming from dimensional reduction of the 4 -form and an extra 2 -form coming from the dualisation of the 4 -form. We denote the corresponding dilaton vectors by $\vec{b}_{i}, \vec{a}_{i j}$ and $\vec{a}$. Note that $\vec{a}$ is given by eqn. (2.6) but with its sign reversed because of the dualisation. The dot products of these vectors are given by eqn. (5.1), together with

$$
\vec{a} \cdot \vec{a}=\frac{5}{2}, \quad \vec{a} \cdot \vec{b}_{i}=-\frac{3}{2}, \quad \vec{a} \cdot \vec{a}_{i j}=\frac{1}{2}, \quad i<j,
$$

The Bogomol'nyi matrix for the elementary case is given by $\mathcal{M}=m \mathbb{1}+p_{i} \Gamma_{0 i}+\frac{1}{2} u_{i j} \Gamma_{0 i j}+u^{*} \Gamma_{\hat{1} \hat{2} \hat{3} \hat{4} \hat{5}}$, where $u^{*}$ is the Page charge for the extra 2-form coming from the dualisation of the 4 -form. The eigenvalues are given by

$$
\begin{aligned}
\mu & =m \pm \sqrt{p_{i} p_{i}+u^{* 2}+\frac{1}{2} u_{i j} u_{i j} \pm \sqrt{X}} \\
X & =4 u_{i j} p_{j} u_{i k} p_{k}+4 p_{i} p_{i} u^{* 2}+\frac{1}{2}\left(u_{i j} u_{i j}\right)^{2}-u_{i j} u_{j k} u_{k l} u_{l i}+\epsilon_{i j k l m} u_{i j} u_{k l} p_{m} u^{*}
\end{aligned}
$$

The eigenvalues for the solitonic case take the same form, with the elementary Page charges replaced by the corresponding solitonic Page charges. 
Now we shall consider $p$-brane solutions with $N \leq 5$ field strengths. When $N=1,2,3$, the solutions are identical to the cases when $D=7$. When $N=4$, in addition to the 3 inequivalent solutions that we discussed in $D=7$, there is an extra inequivalent solution, given by

$$
\Delta=\frac{3}{2}, \quad\left\{p_{1}, u_{12}, u_{13}, u_{45}\right\}=\frac{\lambda}{8}\{1,1,1,1\}, \quad \mu=\frac{\lambda}{4}\left(\sqrt{\frac{8}{3}} \pm \sqrt{1 \pm 1}\right) .
$$

The solution is not supersymmetric.

When $N=5$, there are three inequivalent solutions, given by

$$
\begin{aligned}
\Delta=\frac{12}{5}, & \left\{p_{1}, p_{2}, p_{3}, p_{4}, p_{5}\right\}=\frac{\lambda}{4 \sqrt{5}}\{1,1,1,1,1\} \\
\Delta=\frac{8}{5}, & \left\{p_{1}, u_{12}, u_{14}, u_{23}, u_{45}\right\}=\frac{\lambda}{4 \sqrt{5}}\{1,1,1,1,1\} \\
\Delta=\frac{20}{13}, & \left\{u_{12}, u_{13}, u_{14}, u_{15}, p_{1}\right\}=\frac{\lambda}{4 \sqrt{13}}\{\sqrt{2}, \sqrt{2}, \sqrt{2}, \sqrt{2}, \sqrt{5}\}, .
\end{aligned}
$$

These have eigenvalues $\mu=\frac{\lambda}{4}\left(\sqrt{\frac{5}{3}} \pm 1\right), \mu=\frac{\lambda}{4}\left(\sqrt{\frac{5}{2}} \pm \sqrt{1 \pm \frac{2}{\sqrt{5}}}\right)$ and $\mu=\frac{\lambda}{4}\left(\sqrt{\frac{13}{5}} \pm \sqrt{1 \pm \frac{4 \sqrt{10}}{13}}\right)$ respectively, and so none of these solutions is supersymmetric.

\section{$5.3 \quad D=5$}

In $D=5$, there are six 2 -forms $\mathcal{F}_{M N}^{(i)}$ from the vielbein, fifteen $F_{M N i j}$ coming from dimensional reduction of the 4 -form and six extra 2 -forms coming from the dualisation of the 3 -forms $F_{M N P i}$. We denote the corresponding dilaton vectors by $\vec{b}_{i}, \vec{a}_{i j}$ and $\vec{a}_{i}$. Note that the vectors $\vec{a}_{i}$ are given by eqn. (2.6) but with their signs reversed owing to the dualisation. The dot products of these vectors are given by eqn. (5.1), together with

$$
\begin{aligned}
\vec{a}_{i} \cdot \vec{a}_{j} & =-2 \delta_{i j}+\frac{2}{3}, \quad \vec{a}_{i} \cdot \vec{b}_{j}=2 \delta_{i j}-\frac{4}{3}, \\
\vec{a}_{i} \cdot \vec{a}_{j k} & =-2 \delta_{i j}-2 \delta_{i k}+\frac{2}{3}, \quad j<k,
\end{aligned}
$$

The Bogomol'nyi matrix for the elementary case is given by

$$
\mathcal{M}=m \mathbb{1}+u_{i} \Gamma_{0 i}+\frac{1}{2} u_{i j} \Gamma_{0 i j}+u_{i}^{*} \Gamma_{\hat{1} \hat{2} \hat{3} \hat{4} i}
$$

where $u_{i}^{*}$ are the Page charges for the extra six 2-forms coming from the dualisation of the 3-forms. The general formula for the eigenvalues for this matrix is too complicated to present. We shall instead just give the eigenvalues for the explicit inequivalent solutions that we find. Since the eigenvalues of the Bogomol'nyi matrix for the solitonic case take the identical form, we shall not discuss them further.

In $D=5$, there can be $p$-brane solutions with $N \leq 6$ participating field strengths. The cases with $N=1,2,4$ are precisely the same as in $D=6$, but for $N=3,5$ there is an additional solution 
in each case. Together with five solutions for $N=6$, the full set of new solutions in $D=5$ is

$$
\begin{aligned}
\Delta=\frac{4}{3}, & \left\{u_{12}, u_{34}, u_{56}\right\}=\frac{\lambda}{4 \sqrt{3}}\{1,1,1\} \\
\Delta=\frac{24}{17}, & \left\{u_{12}, u_{13}, u_{45}, u_{56}, u_{46}\right\}=\frac{\lambda}{8 \sqrt{5}}\{2,2, \sqrt{3}, \sqrt{3}, \sqrt{3}\}, \\
\Delta=\frac{7}{3}, & \left\{p_{1}, p_{2}, p_{3}, p_{4}, p_{5}, p_{6}\right\}=\frac{\lambda}{4 \sqrt{6}}\{1,1,1,1,1,1\} \\
\Delta=\frac{3}{2}, & \left\{p_{1}, u_{12}, u_{13}, u_{14}, u_{15}, u_{16}\right\}=\frac{\lambda}{8 \sqrt{2}}\{\sqrt{3}, 1,1,1,1,1\}, \\
\Delta=\frac{15}{11}, & \left\{p_{1}, p_{6}, u_{2}^{*}, u_{3}^{*}, u_{4}^{*}, u_{5}^{*}\right\}=\frac{\lambda}{4 \sqrt{2} 2}\{\sqrt{5}, \sqrt{5}, \sqrt{3}, \sqrt{3}, \sqrt{3}, \sqrt{3}\} \\
\Delta=\frac{7}{5}, & \left\{u_{26}, u_{25}, p_{1}, p_{2}, u_{13}, u_{14}\right\}=\frac{\lambda}{4 \sqrt{10}}\{\sqrt{2}, \sqrt{2}, 1,1, \sqrt{2}, \sqrt{2}\} \\
\Delta=\frac{4}{3}, & \left\{u_{12}, u_{13}, u_{23}, u_{45}, u_{46}, u_{56}\right\}=\frac{\lambda}{4 \sqrt{6}}\{1,1,1,1,1,1\} .
\end{aligned}
$$

All these solutions, except for the case of $\Delta=\frac{4}{3}$ with three field strengths, break all the supersymmetry. From now on, we shall not present the eigenvalues for the non-supersymmetric cases. The eigenvalues for the case of $\Delta=\frac{4}{3}$ with three field strengths are $\frac{2}{3} m\left\{0_{4}, 1_{12}, 2_{12}, 34\right.$. Thus the $p$-brane solution with $\Delta=\frac{4}{3}$ preserves $\frac{1}{8}$ of the supersymmetry. Note that there is also a case with $\Delta=\frac{4}{3}$ for six field strengths, which does not give rise to supersymmetric $p$-brane solutions.

\section{$5.4 D=4$}

In $D=4$, there are a total of twenty-eight 2-form field strengths: seven $\mathcal{F}_{M N}^{(i)}$ from the vielbein, and twenty-one $F_{M N i j}$ coming from dimensional reduction of the 4-form. The corresponding dilaton vectors are denoted by $\vec{b}_{i}$ and $\vec{a}_{i j}$. It turns out that the dot products of these vectors, given by (2.6) and (2.8), are such that we can extend the $i$ index to include the value 8 , and define $\vec{a}_{i 8}=-\vec{b}_{i}$. Then the dot products are given by the single formula

$$
\vec{a}_{i j} \cdot \vec{a}_{k l}=2 \delta_{i k}+2 \delta_{j l}+2 \delta_{i l}+2 \delta_{j k}-1, \quad i<j, k<l,
$$

Although the dilaton vectors can be treated equally by this extension of the index range, the corresponding field strengths do not play equivalent rôles.

In $D=4$, since the dual of a 2 -form is again a 2 -form, in addition to the previous linear combinations of original field strengths we can also combine field strengths and the duals of other field strengths to obtain a $p$-brane solution. Such a phenomenon occurs also in $D=8$ for the 4 -form and $D=6$ for the 3 -forms, as we discussed in the previous section. In terms of the original undualised fields, this corresponds to making an elementary ansatz on some of the fields while making a solitonic ansatz on the remainder. In order to obtain such dyonic solutions, we first dualise certain field strengths, and then use the previously discussed procedure to obtain the Lagrangian (1.1) by taking a linear combination of these together with other undualised field strengths. We 
then make a purely elementary or purely solitonic ansatz on this resulting field strength. The corresponding Page charges of the dualised field strengths are magnetic and electric respectively, precisely opposite to those of the undualised field strengths. As we discussed in section 4.2, these are called dyonic solutions of the first type. We shall discuss dyonic solutions of the second type at the end of the section.

The Bogomol'nyi matrix for all the above mentioned $p$-brane solutions takes the form

$$
\mathcal{M}=m \mathbb{1}+p_{i} \Gamma_{0 i}+\frac{1}{2} u_{i j} \Gamma_{0 i j}+q_{i} \Gamma_{\hat{1} \hat{2} \hat{3} i}+\frac{1}{2} v_{i j} \Gamma_{\hat{1} \hat{2} \hat{3} i j}
$$

An elementary ansatz for an undualised field strength contributes a $p_{i}$ or $u_{i j}$ electric charge, whilst an elementary ansatz for a dualised field strength contributes a $q_{i}$ or $v_{i j}$ magnetic charge. When we present the solutions later in the section, we shall denote such a $q_{i}$ or $v_{i j}$ charge by $p_{i}^{*}$ or $u_{i j}^{*}$. The situation for a solitonic ansatz is the converse of this.

Since there are a total of seven dilatonic scalars in $D=4$, there can be $N \leq 7$ participating field strengths. In general, there can be either purely electric (or purely magnetic), or else mixed dyonic solutions. For $N=1$, the solutions are necessarily either electric or magnetic, with $\Delta=4$, analogous to the cases in $D \geq 5$ that we have discussed previously. All these solutions preserve half the supersymmetry. For $N=2$, there are two different values of $\Delta$ arising from the solutions, namely $\Delta=3$ and $\Delta=2$. Both of these values can be achieved by purely electric (or purely magnetic) solutions. They can also be achieved by mixed dyonic solutions. For higher numbers of field strengths, starting at $N=4$, there are cases that can only be achieved by mixed dyonic configurations; we shall refer to these as intrinsically dyonic solutions. For the purpose of presenting the results, we shall continue with the policy of not giving again solutions that can be obtained from a higher dimension by dimensional reduction, i.e. solutions that have the same $\Delta$ values and the same ratios of participating field strengths. For the new solutions that we do list, we shall favour the purely electric (or purely magnetic) choices where possible, and present dyonic solutions only when they are intrinsically dyonic. We find that the new solutions in $D=4$ are as follows

$$
\begin{aligned}
\Delta=1, & \left\{u_{57}, u_{46}, u_{23}, p_{1}^{*}\right\}=\frac{\lambda}{8}\{1,1,1,1\} \\
\Delta=\frac{6}{5}, & \left\{u_{57}, u_{47}, u_{36}, u_{12}\right\}=\frac{\lambda}{4 \sqrt{10}}\{\sqrt{2}, \sqrt{2}, \sqrt{3}, \sqrt{3}\} \\
\Delta=\frac{12}{11}, & \left\{u_{57}^{*}, u_{47}^{*}, u_{37}, u_{27}, u_{45}\right\}=\frac{\lambda}{4 \sqrt{11}}\{\sqrt{2}, \sqrt{2}, \sqrt{2}, \sqrt{2}, \sqrt{3}\} \\
\Delta=\frac{8}{7}, & \left\{u_{57}, u_{47}, u_{36}, u_{45}, u_{12}\right\}=\frac{\lambda}{4 \sqrt{7}}\{1,1, \sqrt{2}, 1, \sqrt{2}\} \\
\Delta=\frac{20}{17}, & \left\{u_{57}, u_{47}, u_{56}, u_{36}, u_{12}\right\}=\frac{\lambda}{4 \sqrt{17}}\{\sqrt{2}, 2, \sqrt{2}, 2, \sqrt{5}\} \\
\Delta=\frac{10}{9}, & \left\{u_{57}^{*}, u_{47}, u_{37}, u_{27}, u_{17}, u_{56}\right\}=\frac{\lambda}{12 \sqrt{2}}\{\sqrt{5}, \sqrt{2}, \sqrt{2}, \sqrt{2}, \sqrt{2}, \sqrt{5}\} \\
\Delta=\frac{24}{13} & \left\{u_{57}^{*}, u_{47}^{*}, u_{37}, u_{27}, u_{17}, u_{45}\right\}=\frac{\lambda}{4 \sqrt{23}}\{2,2, \sqrt{3}, \sqrt{3}, \sqrt{3}, \sqrt{6}\},
\end{aligned}
$$




$$
\begin{array}{ll}
\Delta=\frac{8}{7}, \quad\left\{u_{57}, u_{47}, u_{56}, u_{36}, u_{34}, u_{12}\right\}=\frac{\lambda}{4 \sqrt{7}}\{1,1,1,1,1, \sqrt{2}\} \\
\Delta=\frac{7}{6}, \quad\left\{u_{57}, u_{47}, u_{37}, u_{56}, u_{24}, u_{13}\right\}=\frac{\lambda}{8 \sqrt{3}}\{1,1,1, \sqrt{3}, \sqrt{3}, \sqrt{3}\} \\
\Delta=\frac{7}{6}, \quad\left\{u_{57}, u_{47}, u_{56}, u_{36}, u_{24}, u_{13}\right\}=\frac{\lambda}{8 \sqrt{3}}\{\sqrt{2}, 1, \sqrt{2}, 1, \sqrt{3}, \sqrt{3}\} \\
\Delta=\frac{3}{2}, \quad\left\{u_{57}, u_{47}, u_{37}, u_{27}, u_{17}, p_{1}\right\}=\frac{\lambda}{8 \sqrt{2}}\{1,1,1,1,1, \sqrt{3}\}, \\
\Delta=\frac{7}{3}, \quad\left\{u_{57}, u_{47}, u_{37}, u_{27}, u_{17}, u_{67}\right\}=\frac{\lambda}{4 \sqrt{6}}\{1,1,1,1,1,1\}, \\
\Delta=\frac{60}{59}, \quad\left\{u_{57}^{*}, u_{47}, u_{37}, u_{27}, u_{17}, u_{56}, u_{67}\right\}=\frac{\lambda}{4 \sqrt{59}}\{\sqrt{10}, \sqrt{6}, \sqrt{6}, \sqrt{6}, \sqrt{6}, \sqrt{15}, \sqrt{10}\} \\
\Delta=\frac{12}{11}, \quad\left\{u_{57}^{*}, u_{47}, u_{37}, u_{27}, u_{17}, u_{56}, p_{6}\right\}=\frac{\lambda}{4 \sqrt{11}}\{\sqrt{3}, 1,1,1,1, \sqrt{3}, 1\}, \\
\Delta=\frac{8}{7}, \quad\left\{u_{57}, u_{47}, u_{56}, u_{36}, u_{24}, u_{13}, u_{12}\right\}=\frac{\lambda}{4 \sqrt{7}}\{1,1,1,1,1,1,1\}, \\
\Delta=\frac{40}{31}, \quad\left\{u_{57}, u_{47}, u_{37}, u_{26}, u_{16}, u_{12}, p_{7}\right\}=\frac{\lambda}{4 \sqrt{31}}\{\sqrt{5}, \sqrt{5}, \sqrt{5}, 2,2,2,2\}, \\
\Delta=\frac{28}{19}, \quad\left\{u_{57}, u_{47}, u_{37}, u_{27}, u_{17}, u_{67}, p_{7}\right\}=\frac{\lambda}{4 \sqrt{19}}\{\sqrt{2}, \sqrt{2}, \sqrt{2}, \sqrt{2}, \sqrt{2}, \sqrt{2}, \sqrt{7}\}, \\
\Delta=\frac{16}{7}, \quad\left\{u_{57}, u_{47}, u_{45}, p_{1}, p_{2}, p_{3}, p_{6}\right\}=\frac{\lambda}{12}\{1,1,1,1,1,1,1\},
\end{array}
$$

where a star on a Page charge indicates that the associated field strength is dualised; these cases are the intrinsically dyonic solutions. Note that all of them satisfy the constraints implied by $\mathcal{L}_{F F A}$ and the Chern-Simons modifications that we discussed in section 2. In fact, this is the first time that we encounter cases where ostensible solutions are actually ruled out by the constraints. For example, the following configurations

$$
\begin{array}{lll}
\Delta=\frac{15}{14}, & \left\{p_{1}^{*}, p_{2}^{*}, p_{3}, u_{45}, u_{23}, u_{36}\right\}=\frac{\lambda}{8 \sqrt{7}}\{\sqrt{6}, \sqrt{3}, \sqrt{5}, \sqrt{5}, \sqrt{3}, \sqrt{6}\}, \\
\Delta=\frac{40}{39}, & \left\{p_{1}^{*}, p_{2}^{*}, p_{3}, u_{45}, u_{56}, u_{23}, u_{37}\right\}=\frac{\lambda}{4 \sqrt{39}}\{\sqrt{8}, \sqrt{4}, \sqrt{5}, \sqrt{5}, \sqrt{5}, \sqrt{4}, \sqrt{8}\}, \\
\Delta=\frac{28}{27}, & \left\{p_{1}, p_{2}^{*}, p_{3}^{*}, u_{45}, u_{46}, u_{23}, u_{17}\right\}=\frac{\lambda}{12 \sqrt{3}}\{\sqrt{2}, 2,2,2,2, \sqrt{2}, \sqrt{7}\}, \\
\Delta=\frac{24}{23}, & \left\{p_{1}^{*}, p_{2}^{*}, u_{34}, u_{35}, u_{26}, u_{67}, u_{17}\right\}=\frac{\lambda}{4 \sqrt{23}}\{\sqrt{3}, \sqrt{3}, 2,2, \sqrt{3}, \sqrt{3}, \sqrt{3}\}, \\
\Delta=\frac{16}{15}, & \left\{p_{1}^{*}, u_{23}, u_{24}, u_{25}, u_{67}, u_{36}, u_{14}\right\}=\frac{\lambda}{4 \sqrt{15}}\{\sqrt{3}, 1,1, \sqrt{3}, \sqrt{3}, \sqrt{2}, \sqrt{2}\} \\
\Delta=\frac{4}{3}, \quad\left\{u_{57}, u_{47}, u_{37}, u_{27}, u_{17}, u_{67}^{*}, p_{7}\right\}=\frac{\lambda}{12}\{1,1,1,1, \sqrt{2}, \sqrt{2}\}
\end{array}
$$

would be solutions were it not for the fact that they do not satisfy the constraint (2.12) implied by the Chern-Simons modifications to the field strengths. This is because in each case, there is an index in common between an undualised 2-form and a dualised 2-form, one coming from the vielbein and the other coming from the field strength $F_{4}$ in $D=11$.

Amongst the new solutions in (5.15), all but the first one, which has $\Delta=1$, break all the supersymmetry. We find that the eigenvalues of the Bogomol'nyi matrix $\mathcal{M}$ for the first solution are given by $m\left(0_{4}, 1_{24}, 2_{4}\right)$, and thus it preserves $\frac{1}{8}$ of the supersymmetry. Together with the solutions that can be obtained from higher dimensions by dimensional reduction, there are a total of four inequivalent supersymmetric particle solutions in $D=4$. They have $\Delta=\frac{4}{N}$ for $N=1,2,3,4$, 
corresponding to $a=\sqrt{3}, 1, \frac{1}{\sqrt{3}}, 0$. (The analogous solutions with the same values of $a$ were also obtained [17] in the four-dimensional $N=4$ heterotic string.) For $N=1,2,3$, the solutions can be purely electric or purely magnetic or mixed dyonic. They preserve $2^{-N}$ of the original $D=11$ supersymmetry. For $N=4$, the solutions are intrinsically dyonic and preserve $\frac{1}{8}$ of the supersymmetry. Although the solutions with $N=3$ and $N=4$ both preserve $\frac{1}{8}$ of the supersymmetry, the degeneracies of the non-zero eigenvalues of their Bogomol'nyi matrices are different. The solutions for $N=1,2,3$ and 4 first appear at $D=10,9,5$ and 4 dimensions respectively. They correspond to what are called stainless super $p$-branes in [9], in the sense that they cannot "oxidise" to isotropic $p$-brane solutions in higher dimensions by the inverse of the dimensional reduction procedure.

So far we have considered either pure elementary or pure solitonic solutions, or else mixed dyonic $p$-brane solutions of the first type, where some of the field strengths are purely elementary and the others are purely solitonic. We now turn to dyonic solutions of the second type, where the canonical field strength has both elementary and solitonic contributions, and hence each participating field strength has the same ratio of electric and magnetic charges. As in the first type of dyonic solution, here too the constraint given in (2.11) must also be satisfied. This is automatic for 2-forms coming from the vielbein; however, if two or more of the 2-forms $F_{2}^{i j}$ coming from $\hat{F}_{4}$ in $D=11$ are involved, then these must each have an index in common with all the others in order to satisfy the constraint. In fact all the previously-listed examples of 2-form field strength configurations can also be used to contruct these dyonic solutions of the second type. However, as we discussed in section 1 , the equations of motion only admit simple solutions when $a=0$ or 1 , corresponding to $\Delta=1$ or 2 . The metrics of these two dyonic particles are given by (1.9) and (1.10) respectively. The corresponding masses, Page charges and the eigenvalues of Bogomol'nyi matrices are given by

$$
\begin{aligned}
& \Delta=1: \quad m=\frac{1}{2} \lambda_{12}, \quad\left\{u_{57}, u_{46}, u_{23}, p_{1}^{*}, v_{57}, v_{46}, v_{23}, q_{1}^{*}\right\}=\frac{1}{8}\left\{\lambda_{1}, \lambda_{1}, \lambda_{1}, \lambda_{1}, \lambda_{2}, \lambda_{2}, \lambda_{2},-\lambda_{2}\right\} \\
& \mu=\frac{1}{2} \lambda_{12}\left\{0_{4}, 1_{24}, 2_{4}\right\} \\
& \Delta=2: \quad m=\frac{\lambda_{1}+\lambda_{2}}{2 \sqrt{2}}, \quad\left\{p_{1}, u_{12}, q_{1}, v_{12}\right\}=\frac{1}{4 \sqrt{2}}\left\{\lambda_{1}, \lambda_{1}, \lambda_{2}, \lambda_{2}\right\} \\
& \mu=\sqrt{2}\left\{\left(\lambda_{1}+\lambda_{2}-\lambda_{12}\right)_{8},\left(\lambda_{1}+\lambda_{2}+\lambda_{12}\right)_{8},\left(\lambda_{1}+\lambda_{2}\right)_{16}\right\}
\end{aligned}
$$

where $\lambda_{12} \equiv \sqrt{\lambda_{1}^{2}+\lambda_{2}^{2}}$. Note that for $\Delta=1$, the solution, is already intrinsically dyonic of the first type even when $\lambda_{1}=0$ or $\lambda_{2}=0$, as we discussed previously. In fact for this solution both the metric and the eigenvalues of the Bogomol'nyi matrix are identical to those of the dyonic solution of the first type, even though the two Bogomol'nyi matrices are different. In particular, the solution always preserves $\frac{1}{4}$ of the supersymmetry, regardless of the values of $\lambda_{1}$ and $\lambda_{2}$. For $\Delta=2$, on 
the other hand, we can have zero eigenvalues only for the following three cases: $\lambda_{1}=0, \lambda_{2}=0$ or $\lambda_{1}=-\lambda_{2}$. The first two cases correspond to the purely solitonic and purely elementary solutions which preserve $\frac{1}{4}$ of the supersymmetry. The third case gives rise to a massless black hole (which has been discussed in 18]), which preserves $\frac{1}{2}$ of the supersymmetry. However, some of the eigenvalues are negative in this case.

\section{$6 \quad p$-branes for 1-form field strengths}

The analysis for the $p$-brane solutions with 1-form field strengths is analogous to that for the higherdegree field strengths. The main difference is that one can only make a solitonic ansatz for a 1-form field strength, since the elementary ansatz (1.4) is not defined when the field strength has only one index. The solitonic solutions are $(D-3)$-branes. In $D \geq 6$, the 1 -forms are given by $F_{M i j k}$ and $\mathcal{F}_{M i}^{(j)}$, and the corresponding solitonic Page charges are denoted by $v_{i j k}$ and $q_{i j}$. In $D=5$, there is an extra 1-form coming from the dualisation of the 4-form $F_{M N P Q}$, whose Page charge is denoted by $v^{*}$. In $D=4$, extra 1 -forms come instead from the dualisation of the seven 3 -forms $F_{M N P i}$, and the corresponding Page charges are denoted by $v_{i}^{*}$. The Bogomol'nyi matrix is given by

$$
\mathcal{M}=m \mathbb{1}+\frac{1}{6} v_{i j k} \Gamma_{\hat{1} \hat{2} i j k}+\frac{1}{2} q_{i j} \Gamma_{\hat{1} \hat{2} i j}+v^{*} \Gamma_{012}+v_{i}^{*} \Gamma_{01 i}
$$

where the $v^{*}$ and $v_{i}^{*}$ terms appear only in $D=5$ and $D=4$ respectively.

The number of $p$-brane solutions for 1-form field strengths is far greater than the previous cases. We shall first present the supersymmetric solutions. Like the $p$-brane solutions for higher-degree field strengths, the solutions with only one participating 1-form field strength also have $\Delta=4$, and they all preserve half the supersymmetry. The first example of such a solution is the solitonic 6-brane in $D=9$ [9], which is the highest dimension for 1-forms. There are a total of 8 inequivalent supersymmetric solutions, given by

$$
\begin{array}{ll}
\Delta=4, \quad\left\{q_{12}\right\}, \quad \mu=2 m\left\{0_{16}, 1_{16}\right\}, \\
\Delta=2, \quad\left\{q_{12}, v_{123}\right\}, \quad \mu=m\left\{0_{8}, 1_{16}, 2_{8}\right\}, \\
\Delta=\frac{4}{3}, \quad\left\{q_{12}, q_{45}, v_{123}\right\}, \quad \mu=\frac{2}{3} m\left\{0_{4}, 1_{12}, 2_{12}, 3_{4}\right\}, \\
\Delta=1, \quad\left\{q_{12}, q_{45}, v_{123}, v_{345}\right\}, \quad \mu=m\left\{0_{4}, 1_{24}, 2_{4}\right\}, \\
\Delta=1, \quad\left\{q_{12}, q_{34}, q_{56}, v_{127}\right\}, \quad \mu=\frac{1}{2} m\left\{0_{2}, 1_{8}, 2_{12}, 3_{8}, 4_{2}\right\}, \\
\Delta=\frac{4}{5}, \quad\left\{q_{12}, q_{34}, q_{56}, v_{127}, v_{347}\right\}, \quad \mu=\frac{2}{5} m\left\{0_{2}, 1_{2}, 2_{12}, 3_{12}, 4_{2}, 5_{2}\right\} \\
\Delta=\frac{2}{3}, \quad\left\{q_{12}, q_{34}, q_{56}, v_{127}, v_{347}, v_{567}\right\}, \quad \mu=\frac{1}{3} m\left\{0_{2}, 2_{6}, 3_{16}, 4_{6}, 6_{2}\right\}, \\
\Delta=\frac{4}{7}, \quad\left\{q_{12}, q_{34}, q_{56}, v_{127}, v_{347}, v_{567}, v_{7}^{*}\right\}, \quad \mu=\frac{2}{7} m\left\{0_{2}, 3_{14}, 4_{14}, 7_{2}\right\} .
\end{array}
$$


It is easy to verify that all these solutions satisfy the constraints implied by both the $\mathcal{L}_{F F A}$ term and the Chern-Simons modifications to the field strengths. The squares of the Page charges listed above are all equal in each case. The first case occurs in $D \leq 9$, and preserves $\frac{1}{2}$ the supersymmetry; the second occurs in $D \leq 8$, and preserves $\frac{1}{4}$ of the supersymmetry. The third and the fourth both occur in $D \leq 6$. They both preserve $\frac{1}{8}$ of the supersymmetry, but their non-vanishing eigenvalues are different. The last four solutions all occur in $D=4$ only, and they all preserve $\frac{1}{16}$ of the supersymmetry, but their non-vanishing eigenvalues are different. Note that we have two cases with $\Delta=1$, with one preserving $\frac{1}{8}$ of the supersymmetry, and the other preserving $\frac{1}{16}$ instead. Some analogous solutions in the four dimensional $N=4$ heterotic string were obtained in [12].

There are a great number of non-supersymmetric solutions. For example for $N=2$ participating field strengths, in addition to the supersymmetric solution with $\Delta=2$, non-supersymmetric solutions exist with $\Delta=1,3$. Both occur in $D \leq 8$. For $N=3$ field strengths, $\Delta=\frac{8}{3}, \frac{12}{7}, \frac{4}{5}, \frac{8}{11}, \frac{2}{5}$, with the first two occurring in $D \leq 7$ and the additional ones occurring only when $D \leq 6$. As the number of participating field strengths increases, which occurs as we descend to lower dimensions, the number of non-supersymmetric solutions increases significantly. For example there are 43 different $\Delta$ values for six field strengths in $D=5$. However it is a straightforward exercise to enumerate them by computer, using the procedure discussed in section 3 . It involves solving eqn. (3.4) for all possible $N \times N$ submatrices $A_{\alpha \beta}$ of the dot products of the dilaton vectors, with $N \leq(11-D)$. We shall not present these results in this paper.

\section{Conclusions}

In this paper, we have looked at $p$-brane solutions in the maximal supergravities in $11 \geq D \geq 4$ dimensions. Our starting point was to obtain the complete bosonic Lagrangians via Kaluza-Klein dimensional reduction from $D=11$. Our construction of the Lagrangians distinguishes the $(11-D)$ dilatonic scalar fields from the 0 -form potentials for the 1 -form field strengths. We then consistently truncate the bosonic Lagrangian to the form (1.1), with one dilatonic scalar and one $n$-form field strength. The $p$-brane solutions involve only the metric, the dilatonic scalar (which is a linear combination of the original $(11-D)$ dilatonic scalars), and the field strength (which is a linear combination of the original field strengths). We also discussed the further constraints on the $p$ brane solutions implied by the terms coming from the dimensional reduction of $\mathcal{L}_{F F A}$, and by the Chern-Simons modifications of the field strengths, since we focussed only on $p$-brane solutions not involving these terms. Classifying $p$-brane solutions of this kind reduces to a problem in linear algebra, as we discussed in section 3 . 
The metrics of these $p$-brane solutions depend only on the degree of the field strength and the value of $\Delta$ given by (1.3). Different consistent truncations lead to different values of $\Delta$. Another factor that characterises a $p$-brane solution is its supersymmetry. The supersymmetry of a solution depends on both the value of $\Delta$ and the configuration of the participating field strengths. In order to study the supersymmetry of the $p$-brane solutions, we obtained Nester forms in lower dimensions using Kaluza-Klein dimensional reduction from $D=11$. From the Nester form, we obtain the Bogomol'nyi matrix, whose zero eigenvalues correspond to the unbroken supersymmetries. In fact a $p$-brane solution is also characterised by the eigenvalues of its Bogomol'nyi matrix. As we descend through the dimensions, the number of field strengths grows rapidly, and, as a consequence, the number of inequivalent $p$-brane solutions proliferates. However, most of these solutions are nonsupersymmetric, i.e. the corresponding Bogomol'nyi matrices have no zero eigenvalues. Although the solutions with some unbroken supersymmetry are of primary interest, we have also given a fairly exhaustive classification of the non-supersymmetric solutions too. Specifically, we have presented all the inequivalent $p$-brane solutions using 4-form, 3-form and 2-form field strengths in all dimensions $D \geq 4$, and some examples of $p$-brane solutions using 1-form field strengths. For the supersymmetric solutions, on the other hand, we have exhaustively studied all field strengths in all dimensions $\geq 4$.

The values of $\Delta$ for the supersymmetric $p$-brane solutions can be summarised as follows:

\begin{tabular}{|c|c|c|c|c|}
\hline & 4 -form & 3-form & 2-form & 1-form \\
\hline \hline$D=11$ & $\Delta=4$ & & & \\
\hline$D=10$ & & $\Delta=4$ & $\Delta=4$ & \\
\hline$D=9$ & & & $\Delta=2$ & $\Delta=4$ \\
\hline$D=8$ & & & & $\Delta=2$ \\
\hline$D=7$ & & & & \\
\hline$D=6$ & & $\Delta=2$ & & $\Delta=\frac{4}{3}, 1^{\prime}$ \\
\hline$D=5$ & & & $\Delta=\frac{4}{3}$ & \\
\hline$D=4$ & & & $\Delta=1^{\prime}$ & $\Delta=1, \frac{4}{5}, \frac{2}{3}, \frac{4}{7}$ \\
\hline
\end{tabular}

Table 2: Supersymmetric $p$-brane solutions

Note that we present the the values of $\Delta$ in the dimensions where $p$-brane solutions using the indicated field strengths first arise. Supersymmetric solutions with the same values of $\Delta$ then occur in all lower dimensions. Note also that in a lower dimension, higher-degree field strengths can be dualised to lower-degree field strengths. All these supersymmetric solutions have $\Delta=\frac{4}{N}$, where $N$ is the number of field strengths participating in the solution. The number of different 
values of $\Delta$ increases as the degree of the participating field strength decreases. The Page charges for all these fields are equal in each given supersymmetric solution; denoting this value by $P$, we find that it is related to the mass per unit volume $m$ by

$$
\frac{m}{P}=N
$$

The supersymmetric $p$-brane solutions with $\Delta=4,2$ and $\frac{4}{3}$ preserve $\frac{1}{2}, \frac{1}{4}$ and $\frac{1}{8}$ of the $D=11$ supersymmetry respectively. The eigenvalues of their Bogomol'nyi matrices are $2 m\left\{0_{16}, 1_{16}\right\}$, $m\left\{0_{8}, 1_{16}, 2_{8}\right\}$ and $\frac{2}{3} m\left\{0_{4}, 1_{12}, 2_{12}, 3_{4}\right\}$. For $\Delta=1$, there are two inequivalent supersymmetry breaking patterns, which we distinguish by writing them as $\Delta=1$ and $\Delta=1^{\prime}$. Both the solution in $D=4$ using 2 -forms and the solution in $D=6$ using 1-forms, i.e. $\Delta=1^{\prime}$, preserve $\frac{1}{8}$ of the supersymmetry, and the eigenvalues are $m\left\{0_{4}, 1_{24}, 2_{4}\right\}$. On the other hand, the solution in $D=4$ using 1 -forms, i.e. $\Delta=1$, has eigenvalues $\frac{1}{2} m\left\{0_{2}, 1_{8}, 2_{12}, 3_{8}, 4_{2}\right\}$ and hence preserves $\frac{1}{16}$ of the supersymmetry. The remaining three cases, with $\Delta=\frac{4}{5}, \frac{2}{3}$ and $\frac{4}{7}$ for 1 -forms in $D=4$, all preserve $\frac{1}{16}$ of the supersymmetry, and have eigenvalues equal to $\frac{2}{5} m\left\{0_{2}, 1_{2}, 2_{12}, 3_{12}, 4_{2}, 5_{2}\right\}, \frac{1}{3} m\left\{0_{2}, 2_{6}, 3_{16}, 4_{6}, 6_{2}\right\}$ and $\frac{2}{7} m\left\{0_{2}, 3_{14}, 4_{14}, 7_{2}\right\}$ respectively. It is worth remarking also that in the cases of $\Delta=4,2, \frac{4}{3}$ and 1, the eigenvalues of the Bogomol'nyi matrices, and hence the supersymmetries, are independent of the signs of the Page charges. On the other hand, in the cases of $\Delta=1^{\prime}, \frac{4}{5}, \frac{2}{3}$ and $\frac{4}{7}$, there are two inequivalent sets of eigenvalues depending on the signs of the Page charges, one of which (the one we have presented) includes zero eigenvalues, whilst the other does not.

The above supersymmetric $p$-branes are either purely elementary or purely solitonic, or else mixed dyonic of the first type. The characteristic feature of these solutions is that each participating field strength has either elementary or solitonic (but not both) contributions. For the 3-forms in $D=6$ and 2-forms in $D=4$, we can also construct dyonic solutions of the second type, where the canonical field strength has both elementary and solitonic contributions, and hence each participating field strength has the same ratio of electric and magnetic Page charges. In $D=6$, the supersymmetric dyonic string preserves $\frac{1}{4}$ of the supersymmetry for generic non-vanishing electric and magnetic charges. The mass per unit length is the sum of the electric and magnetic charges. When one of the Page charges is set to zero, the solution reduces to the purely elementary or purely solitonic solution. When the two Page charges are equal, the solution reduces to the selfdual string in $D=6$ self-dual supergravity. On the other hand, when the two Page charges are equal and opposite, the solution, although anti-self-dual, does not reduce to the anti-self-dual string in anti-self-dual $D=6$ supergravity, but instead gives rise to a massless string, however with a Bogomol'nyi matrix of indefinite signature. Dyonic solutions of the second type also arise in $D=4$. The solutions are supersymmetric for $\Delta=1$ and 2. For $\Delta=1$, the solution preserves 
$\frac{1}{4}$ of the supersymmetry; for $\Delta=2$, it gives rise to a massless black hole that preserves $\frac{1}{2}$ of the supersymmetry.

\section{Appendix}

In this appendix, we present the complete bosonic Lagrangian for maximal supergravity in $D$ dimensions, obtained by dimensional reduction from $D=11$. Thus starting from the Lagrangian (2.9), we apply the reduction procedure described in section 2 , leading to

$$
\begin{aligned}
\mathcal{L}= & e R-\frac{1}{2} e(\partial \vec{\phi})^{2}-\frac{1}{48} e e^{\vec{a} \cdot \vec{\phi}} F_{4}^{2}-\frac{1}{12} e \sum_{i} e^{\vec{a}_{i} \cdot \vec{\phi}}\left(F_{3}^{i}\right)^{2}-\frac{1}{4} e \sum_{i<j} e^{\vec{a}_{i j} \cdot \vec{\phi}}\left(F_{2}^{i j}\right)^{2} \\
& -\frac{1}{4} e \sum_{i} e^{\vec{b}_{i} \cdot \vec{\phi}}\left(\mathcal{F}_{2}^{i}\right)^{2}-\frac{1}{2} e \sum_{i<j<k} e^{\vec{a}_{i j k} \cdot \vec{\phi}}\left(F_{1}^{i j k}\right)^{2}-\frac{1}{2} e \sum_{i<j} e^{\vec{b}_{i j} \cdot \vec{\phi}}\left(\mathcal{F}_{1}^{i j}\right)^{2}+\mathcal{L}_{F F A},
\end{aligned}
$$

where the dilaton vectors are defined by (2.6) and $\mathcal{L}_{F F A}$ is coming from the $\hat{F}_{4} \wedge \hat{F}_{4} \wedge \hat{A}_{3}$ term in the $D=11$ Lagrangian. We shall discuss this term presently.

First we discuss the Chern-Simons modifications to the various field strengths appearing in (A.1). The Kaluza-Klein reduction of an $n$-form potential $\hat{A}_{n}$ from $D+1$ to $D$ dimensions is given by $\hat{A}_{n}=A_{n}+A_{n-1} \wedge d z$, and so the 3 -form potential of $D=11$ supergravity, when reduced to $D$ dimensions, is given by

$$
\hat{A}_{3}=A_{3}+A_{2}^{i} \wedge d z^{i}-\frac{1}{2} A_{1}^{i j} \wedge d z^{i} \wedge d z^{j}-\frac{1}{6} A_{0}^{i j k} d z^{i} \wedge d z^{j} \wedge d z^{k}
$$

Taking the exterior derivative, we have

$$
\hat{F}_{4}=\tilde{F}_{4}+\tilde{F}_{3}^{i} \wedge d z^{i}-\frac{1}{2} \tilde{F}_{2}^{i j} \wedge d z^{i} \wedge d z^{j}-\frac{1}{6} \tilde{F}_{1}^{i j k} \wedge d z^{i} \wedge d z^{j} \wedge d z^{k}
$$

where $\tilde{F}_{4}=d A_{3}, \tilde{F}_{2}^{i j}=d A_{1}^{i j}$ and $\tilde{F}_{1}^{i j k}=d A_{0}^{i j k}$ are the unmodified field strengths. In order to adapt the expansion (A.3) to the vielbein basis, we re-express $d z^{i}$ in terms of $h^{i}=d z^{i}+\mathcal{A}_{1}^{i}+\mathcal{A}_{0}^{i j} d z^{j}$, which is the subsequent dimensional reduction of the term $\left(d z^{i}+\mathcal{A}_{1}^{i}\right)$ arising in the Kaluza-Klein vielbein ansatz at the $i$ 'th step of the reduction process. Since the sum in the last term is only over $j>i$, it is easy to solve iteratively for $d z^{i}$, giving

$$
\begin{aligned}
d z^{i} & =\left(\delta^{i \ell}-\mathcal{A}_{0}^{i \ell}+\mathcal{A}_{0}^{i j} \mathcal{A}_{0}^{j \ell}-\mathcal{A}_{0}^{i j} \mathcal{A}_{0}^{j k} \mathcal{A}_{0}^{k \ell}+\cdots\right)\left(h^{\ell}-\mathcal{A}_{1}^{\ell}\right) \\
& =\left[\left(1+\mathcal{A}_{0}\right)^{-1}\right]^{i \ell}\left(h^{\ell}-\mathcal{A}_{1}^{\ell}\right) \\
& \equiv \gamma^{i \ell}\left(h^{\ell}-\mathcal{A}_{1}^{\ell}\right)
\end{aligned}
$$

Since the 0 -form potentials $\mathcal{A}_{0}^{i j}$ are defined only for $i<j$, i.e. $\mathcal{A}_{0}^{i j}=0$ for $i \geq j$, it follows that the binomial expansion of $\left[\left(1+\mathcal{A}_{0}\right)^{-1}\right]^{i \ell}$ terminates with the power $\left(\mathcal{A}_{0}\right)^{10-D}$ in $D$ dimensions. Also, 
we have $\gamma^{i j}=0$ for $i>j$ and $\gamma^{i j}=1$ for $i=j$. Thus the 11-dimensional 4 -form field strength A.3) can be rewritten as

$$
\hat{F}_{4}=F_{4}+F_{3}^{i} \wedge h^{i}-\frac{1}{2} F_{2}^{i j} \wedge h^{i} \wedge h^{j}-\frac{1}{6} F_{1}^{i j k} \wedge h^{i} \wedge h^{j} \wedge h^{k}
$$

where the Chern-Simons modified field strengths, denoted by untilded symbols, are given by

$$
\begin{aligned}
F_{4} & =\tilde{F}_{4}-\gamma^{i j} \tilde{F}_{3}^{i} \wedge \mathcal{A}_{1}^{j}-\frac{1}{2} \gamma^{i k} \gamma^{j \ell} \tilde{F}_{2}^{i j} \wedge \mathcal{A}_{1}^{k} \wedge \mathcal{A}_{1}^{\ell}+\frac{1}{6} \gamma^{i \ell} \gamma^{j m} \gamma^{k n} \tilde{F}_{1}^{i j k} \wedge \mathcal{A}_{1}^{\ell} \wedge \mathcal{A}_{1}^{m} \wedge \mathcal{A}_{1}^{n} \\
F_{3}^{i} & =\gamma^{j i} \tilde{F}_{3}^{j}-\gamma^{j i} \gamma^{k \ell} \tilde{F}_{2}^{j k} \wedge \mathcal{A}_{1}^{\ell}-\frac{1}{2} \gamma^{j i} \gamma^{k m} \gamma^{\ell n} \tilde{F}_{1}^{j k \ell} \wedge \mathcal{A}_{1}^{m} \wedge \mathcal{A}_{1}^{n}, \\
F_{2}^{i j} & =\gamma^{k i} \gamma^{\ell j} \tilde{F}_{2}^{k \ell}-\gamma^{k i} \gamma^{\ell j} \gamma^{m n} \tilde{F}_{1}^{k \ell m} \wedge \mathcal{A}_{1}^{n}, \\
F_{1}^{i j k} & =\gamma^{\ell i} \gamma^{m j} \gamma^{n k} \tilde{F}_{1}^{\ell m n} .
\end{aligned}
$$

These modified field strengths are the ones that appear in the kinetic terms in the Lagrangian (A.1). Similarly, the Chern-Simons modifications to the 2-forms and 1-forms coming from the vielbein are given by

$$
\begin{aligned}
\mathcal{F}_{2}^{i} & =\tilde{\mathcal{F}}_{2}^{i}-\gamma^{j k} \tilde{\mathcal{F}}_{1}^{i j} \wedge \mathcal{A}_{1}^{k}, \\
\mathcal{F}_{1}^{i j} & =\gamma^{k j} \tilde{\mathcal{F}}_{1}^{i k},
\end{aligned}
$$

where again the tildes denote the unmodified field strengths; $\tilde{\mathcal{F}}_{2}^{i}=d \mathcal{A}_{1}^{i}, \tilde{\mathcal{F}}_{1}^{i j}=d \mathcal{A}_{0}^{i j}$. Note that all the Chern-Simons modifications become much simpler if the 0 -form potentials $\mathcal{A}_{0}^{i j}$ from the vielbein vanish, since then $\gamma^{i j}$ is simply $\delta^{i j}$.

Let us now consider the dimensional reduction of the $\hat{F}_{4} \wedge \hat{F}_{4} \wedge \hat{A}_{3}$ term in the $D=11$ Lagrangian. Since this term is constructed without the use of the metric or vielbein, its dimensional reduction is written in terms of the unmodified field strengths $\tilde{F}_{4}, \tilde{F}_{3}^{i}, \tilde{F}_{2}^{i j}$ and $\tilde{F}_{1}^{i j k}$. Thus from (A.2) and A.3) we find that the term $\mathcal{L}_{F F A}$ is dimensionally reduced to

$$
\begin{array}{ll}
D=10: & \frac{1}{2} \tilde{F}_{4} \wedge \tilde{F}_{4} \wedge A_{2}, \\
D=9: & \left(-\frac{1}{4} \tilde{F}_{4} \wedge \tilde{F}_{4} \wedge A_{1}^{i j}-\frac{1}{2} \tilde{F}_{3}^{i} \wedge \tilde{F}_{3}^{j} \wedge A_{3}\right) \epsilon_{i j} \\
D=8: & \left(-\frac{1}{12} \tilde{F}_{4} \wedge \tilde{F}_{4} A_{0}^{i j k}-\frac{1}{6} \tilde{F}_{3}^{i} \wedge \tilde{F}_{3}^{j} \wedge A_{2}^{k}+\frac{1}{2} \tilde{F}_{3}^{i} \wedge \tilde{F}_{2}^{j k} \wedge A_{3}\right) \epsilon_{i j k}, \\
D=7: & \left(-\frac{1}{6} \tilde{F}_{4} \wedge \tilde{F}_{3}^{i} A_{0}^{j k l}+\frac{1}{6} \tilde{F}_{3}^{i} \wedge \tilde{F}_{3}^{j} \wedge A_{1}^{k l}+\frac{1}{8} \tilde{F}_{2}^{i j} \wedge \tilde{F}_{2}^{k l} \wedge A_{3}\right) \epsilon_{i j k l}, \\
D=6: & \left(\frac{1}{12} \tilde{F}_{4} \wedge \tilde{F}_{2}^{i j} A_{0}^{k l m}+\frac{1}{12} \tilde{F}_{3}^{i} \wedge \tilde{F}_{3}^{j} A_{0}^{k l m}+\frac{1}{8} \tilde{F}_{2}^{i j} \wedge \tilde{F}_{2}^{k l} \wedge A_{2}^{m}\right) \epsilon_{i j k l m}, \\
D=5: & \left(\frac{1}{12} \tilde{F}_{3}^{i} \wedge \tilde{F}_{2}^{j k} A_{0}^{l m n}-\frac{1}{48} \tilde{F}_{2}^{i j} \wedge \tilde{F}_{2}^{k l} \wedge A_{1}^{m n}-\frac{1}{72} \tilde{F}_{1}^{i j k} \wedge \tilde{F}_{1}^{l m n} \wedge A_{3}\right) \epsilon_{i j k l m n}, \\
D=4: & \left(-\frac{1}{48} \tilde{F}_{2}^{i j} \wedge \tilde{F}_{2}^{k l} A_{0}^{m n p}-\frac{1}{72} \tilde{F}_{1}^{i j k} \wedge \tilde{F}_{1}^{l m n} \wedge A_{2}^{p}\right) \epsilon_{i j k l m n p}, \\
D=3: & \frac{1}{144} \tilde{F}_{1}^{i j k} \wedge \tilde{F}_{1}^{l m n} \wedge A_{1}^{p q} \epsilon_{i j k l m n p q}, \\
D=2: & \frac{1}{1296} \tilde{F}_{1}^{i j k} \wedge \tilde{F}_{1}^{l m n} A_{0}^{p q r} \epsilon_{i j k l m n p q r} .
\end{array}
$$


In the $p$-brane solutions that we are considering in this paper, the contributions that these terms give to the field equations will always be required to be zero. This leads to the constraints (2.11) discussed in section 2 .

\section{Acknowledgement}

We should like to thank M.J. Duff, J. Rahmfeld and E. Sezgin for useful discussions.

\section{References}

[1] A. Dabholkar, G.W. Gibbons, J.A. Harvey and F. Ruiz Ruiz, Nucl. Phys. B340 (1990) 33.

[2] M.J. Duff and K.S. Stelle, Phys. Lett. B253 (1991) 113.

[3] M.J. Duff and J.X. Lu, Nucl. Phys. B354 (1991) 141. R.R. Khuri, Phys. Lett. 307 (1993) 302; R.R. Khuri, Nucl. Phys. B403 (1993) 335;

[4] C.G. Callan, J.A. Harvey and A. Strominger, Nucl. Phys. B359 (1991) 611; Nucl. Phys. B367 (1991) 60; Supersymmetric String Solitons, Lectures given at the Trieste Summer School, Trieste, Italy, 1991.

[5] A. Strominger, Nucl. Phys. B343 (1990) 167; M.J. Duff and J.X. Lu, Phys. Rev. Lett. 66 (1991) 1402.

[6] M.J. Duff, R.R. Khuri and J.X. Lu, Phys. Rep. 259 (1995) 213.

[7] R. Güven, Phys. Lett. B276 (1992) 49; Phys. Lett. B212 (1988) 277.

[8] M.J. Duff and J.X. Lu, Phys. Lett. B273 (1991) 409.

[9] H. Lü, C.N. Pope, E. Sezgin and K.S. Stelle, Stainless super p-branes, CTP TAMU-31/95, Imperial/TP/94-95/56, SISSA 97/95/EP, hep-th/9508042, to appear in Nucl. Phys. B.

[10] J. Polchinski and E. Witten, Evidence for heterotic type I string duality, hep-th/9510169.

[11] H. Lü, C.N. Pope, E. Sezgin and K.S. Stelle, Dilatonic p-brane solitons, CTP-TAMU-40/95, Imperial/TP/95-96/11, hep-th/9511203.

[12] M.J. Duff, A. Ferrara, R.R. Khuri and J. Rahmfeld, Phys. Lett. B356 (1995) 479.

[13] E. Cremmer, B. Julia and J. Scherk, Phys. Lett. B76 (1978) 409. 
[14] J.M. Izquierdo, N.D. Lambert, G. Papadopoulos and P.K. Townsend, Dyonic membranes, DAMTP-R-95-40, hep-th/9508177.

[15] D.N. Page, Phys. Rev. D28 (1983) 2976.

[16] L.J. Romans, Nucl. Phys. B276 (1986) 71.

[17] M.J. Duff and J. Rahmfeld, Phys. Lett. B345 (1995) 441; M.J. Duff, J.T. Liu and J. Rahmfeld, Four-dimensional string/string/string triality, preprint CTP-TAMU-27/95, hep-th/9508094.

[18] A. Strominger, Nucl. Phys. B451 (1995) 96; M. Cvetic and D. Youm, Phys. Lett. B359 (1995) 87; K. Behrndt, About a class of exact string backgrounds, preprint HUB-EP-95/6, hep-th/9506106; R.Kallosh and A. Linde, Exact supersymmetric massive and massless white holes, SU-ITP-95-14, hep-th/9507022. 